\title{
REMARKS ON THE THEOREM OF ELLIOTT AND DABOUSSI, AND APPLICATIONS*
}

\author{
WOLFGANG SCHWARZ \\ Fachbereich Mathematik, Johann Wolfgang Goethe Universität \\ Frankfurt am Main, F.R.G.
}

\section{Introduction and motivation}

\subsection{Mean values}

Very often, not only in analytic number theory but also in algebra, combinatorics, geometry and in other fields it is an important task to decide whether an arithmetical function

$$
f: N \rightarrow C
$$

has a mean value

$$
M(f)=\lim _{x \rightarrow \infty} \frac{1}{x} \sum_{n \leqslant x} f(n),
$$

and to calculate this limit. (1)

For example, results on the distribution of primes, on the distribution of squarefree or $k$-free numbers, on lattice points in certain regions, on the

* Extended version of two survey lectures given at the Warszawa International Banach Center on September 6, 1982 and September 8, 1982.

(1) Often the calculation of this limit is not too difficult; by partial summation the existence of $M(f)$ implies

$$
F(s):=\sum_{n=1}^{\infty} \frac{f(n)}{n^{s}}=s \int_{1}^{\infty} \sum_{n<u} f(n) \frac{d u}{u^{s+1}} \sim M(f) \frac{s}{s-1}
$$

for $s \rightarrow 1$, Re $s>1$. Hence

$$
M(f)=\lim _{s \rightarrow 1^{+}}(s-1) F(s),
$$

if the existence of $M(f)$ is known in advance. 
density of subsets of integers, on counting functions for algebraic objects, and other results may be described as results on the existence of mean values for certain arithmetical functions:

- $M(\mu)=0$ (for the Möbius function $\mu$ ) is equivalent to the prime number theorem,

- $M\left(\mu^{2}\right)=6 / \pi^{2}$, and

$$
M\left(\mu^{2} \chi_{a \bmod b}\right)=\frac{6}{\pi^{2}} \cdot \frac{1}{b} \cdot \prod_{p \mid b}\left(1-p^{-2}\right)^{-1} \prod_{p\left|(a, b),\left(p^{2}, b\right)\right| a}\left(1-\frac{\left(p^{2}, b\right)}{p^{2}}\right)
$$

(where $\chi_{a \text { modb }}$ is the characteristic function of the set of integers $n \equiv a \bmod b$ ) give information on the distribution of squarefree numbers,

- $M(r)=\pi$ and $M(\tau)=\infty$ (where $r(n)$ is the number of representations of $n$ as a sum of two squares and $\tau(n)$ the divisor function) are results on the number of lattice points in circles or hyperbolas,

- the formula $M(a)=\prod_{v=2}^{\infty} \zeta(v)$, where $a(n)$ is the number of (nonisomorphic) abelian groups of order $n$, is related to certain algebraic objects,

- the existence of $M\left(\varrho_{P}\right)$ for $\varrho_{P}(n)$ counting the solutions of the polynomial congruence $P(d) \equiv 0 \bmod n$, where $P(X) \in Z[X]$ is irreducible, is a rather deep result in number theory. ${ }^{(2)}$

Further interesting examples of mean values are the Fourier coefficients

$$
\hat{f}(\alpha)=M\left(f \bar{e}_{\alpha}\right), \quad \text { where } e_{\alpha}(n)=e^{2 \pi i a n},
$$

and the Ramanujan coefficients ${ }^{(3)}$

$$
a_{r}(f)=\frac{1}{\varphi(r)} M\left(f c_{r}\right)
$$

of arithmetical functions.

According to $\mathrm{H}$. Weyl's criterion [79] a sequence $\left\{x_{n}\right\}_{n=1,2, \ldots}$ of real numbers is uniformly distributed modulo one, if the mean values

$$
M\left(n \mapsto \exp \left\{2 \pi i k x_{n}\right\}\right)
$$

are zero for any integer $k \neq 0$.

The problem of the existence of a limit-distribution for real-valued

(2) This follows for example from Wirsing's theorem (Section 1.2) in combination with Lemma 7 from Erdos [25].

(3) $c_{r}$ denotes the Ramanujan sum, defined by

$$
c_{r}(n)=\sum_{d \mid(n, r)} d \cdot \mu\left(\frac{r}{d}\right)=\sum_{\substack{1<a \leq r \\(a, r)=1}} \exp \left(2 \pi i \frac{a}{r} n\right) .
$$


arithmetical functions $g$,

$$
\lim _{N \rightarrow \infty} \frac{1}{N} \#\{n \leqslant N, g(n) \leqslant x\}=\Psi(x)
$$

is closely connected with the existence of

$$
M(n \mapsto \exp \{2 \pi i t g(n)\})
$$

for any real $t$, according to the continuity theorem for characteristic functions (see for example E. Lukacs [54], Theorem 3.6.1).

\subsection{Multiplicative functions}

The property "multiplicativity" of arithmetical functions can be defined by the requirements

$$
\left.f(n)=\prod_{p^{k} \| n} f\left(p^{k}\right) \quad \text { (for any } n\right) \quad \text { and } \quad f(1)=1,
$$

and thus these functions are determined by their values at the prime powers. Higher prime-powers are "rare", hence it may be expected that the behaviour of $f$ in the mean is determined by the behaviour of $f$ at the primes. Another formulation of this principle is contained in the relations

$$
\sum_{n=1}^{\infty} \frac{f(n)}{n^{s}}=\prod_{p}\left\{1+\frac{f(p)}{p^{s}}+\frac{f\left(p^{2}\right)}{p^{2 s}}+\ldots\right\} \sim C_{f} \prod_{p}\left(1+\frac{f(p)}{p^{s}}\right) \sim C_{f}^{*} \exp \left(\sum_{p} \frac{f(p)}{p^{s}}\right)
$$

for $s \rightarrow 1+$, under suitable conditions on the multiplicative function $f$.

More precisely: (4) If $f$ is multiplicative, $f(p) \geqslant c_{1}>0,0 \leqslant f\left(p^{k}\right) \leqslant c_{2}$, then

$$
\sum_{n \leqslant x} f(n) \bigcup x \exp \left\{\sum_{p \leqslant x} \frac{1}{p}(f(p)-1)\right\} .
$$

The above-mentioned idea was very clearly expressed by Eduard Wirsing, and he was able to transform this idea into a rather general theorem on the behaviour of sums $\sum_{n \leqslant x} f(n)$ for nonnegative multiplicative functions, a theorem, which has a lot of applications.

THEOREM (E. Wirsing [81]). For nonnegative multiplicative functions $f$ with "small values" at higher prime-powers, ${ }^{(5)}$ satisfying

$$
\sum_{p \leqslant x} f(p) \log p \sim \tau x \quad(x \rightarrow \infty), \quad \tau>0,
$$

(4) See Barban [1], Theorem 8.1, and Wolke [86], Theorem 1 and 2 for more general results. See also G. Halász [30], Theorem 3. For a weaker, but very useful result, see Lemma 5.5.

(5) This means $0 \leqslant f\left(p^{k}\right) \leqslant \gamma_{1} \cdot \gamma_{2}^{k}$ for $k \geqslant 2$, where $\gamma_{2}<2$. 
the asymptotic relation

$$
\sum_{n \leqslant x} f(n)=(1+o(1)) \frac{e^{-\varphi_{p}}}{\Gamma(\tau)} \cdot \frac{x}{\log x} \prod_{p \leqslant x}\left(1+\frac{f(p)}{p}+\frac{f\left(p^{2}\right)}{p^{2}}+\ldots\right)
$$

is true, where $\mathscr{E}=0.577 \ldots$ is Euler's constant. ${ }^{(6)}$

About the same time $H$. Delange [12] gave another result, which is concerned with multiplicative functions of absolute value $|f| \leqslant 1$ only, but gives necessary and sufficient conditions for the existence of a non-zero mean value.

Theorem of Delange. For multiplicative functions $f: N \rightarrow C$ satisfying $|f| \leqslant 1$ the following conditions are equivalent.

$\left(\mathrm{D}_{1}\right)$ The mean value $M(f)$ exists and is non-zero.

$\left(D_{2}\right)$ The series

$$
S_{1}(f)=\sum_{p} \frac{1}{p}\{f(p)-1\}
$$

is convergent, and for all primes $p$

$$
\sum_{k=0}^{\infty} \frac{1}{p^{k}} f\left(p^{k}\right) \neq 0 .{ }^{(7)(8)}
$$

(6) Wirsing's paper aiso contains interesting results on complex-valued multiplicative functions. The important paper Wirsing [82] is concerned with weakening condition (1.5), assuming only an asymptotic formula for

$$
\sum_{p \leqslant x} \frac{1}{p} f(p) \log p .
$$

One of his results is the following: Let $f \geqslant 0$ be multiplicative, assume $f(p) \leqslant \gamma_{3}$ for all primes $p$, and

$$
\sum_{p} \sum_{k \geqslant 2} p^{-k} f\left(p^{k}\right)<\infty
$$

If

$$
\left.\sum_{p \leqslant x} \frac{\log p}{p} f(p) \sim \tau \log x \quad \text { (with } \tau>0\right)
$$

and

$$
\sum_{p} \sum_{k \geqslant 2} f\left(p^{k}\right) \ll x(\log x)^{-1} \quad(\text { if } \tau \leqslant 1),
$$

then formula (1.6) is true.

(7) Since $|f| \leqslant 1$, condition (1.8) is trivially satisfied for any prime $p>2$.

(8) If $M(f)$ exists, then by partial summation

$$
M(f)=\lim _{\sigma \rightarrow 1+} \prod_{p}\left(1+\frac{f(p)}{p^{\sigma}}+\frac{f\left(p^{2}\right)}{p^{2 \sigma}}+\ldots\right)\left(1-\frac{1}{p^{\sigma}}\right) .
$$

Thus condition (1.8) excludes the possibility that $M(f)=0$ trivially by the vanishing of a factor in the product representation (1.9). 
Thus in order to have a non-zero mean value, a multiplicative function $f$ must have its values $f(p)$ mostly near 1 .

\subsection{The theorem of Halász on mean values of arithmetical functions}

In this survey, results on multiplicative functions which might have a mean value zero will not be treated. However we have to mention the deep results of E. Wirsing [82] ${ }^{(9)}$ and G. Halász [28]. The latter used the classical method of complex integration in a very skilful and sophisticated way to prove the

THEOREM OF HALÁSZ. If $f: N \rightarrow C$ is multiplicative and $|f| \leqslant 1$, then there are constants $C \in C, a \in R$ and a slowly oscillating ${ }^{(10)}$ function $L$ of modulus $|L|=1$, such that

$$
\sum_{n \leqslant x} f(n)=C(1+o(1)) x^{1+i a} L(\log x) .
$$

In the case of $f$ being real-valued $a$ and $L$ are given by $a=0$ and $L$ $=1$, that means: $M(f)$ exists for any real-valued multiplicative function of modulus at most $1 .^{(11)}$

The mean value $M(f)$ is zero, if

$$
\Sigma(t):=\sum_{p} \frac{1}{p}\left(1-\operatorname{Re}\left\{f(p) p^{-i t}\right\}\right)=\infty
$$

for all $t \in \boldsymbol{R}$. If there is a real number $t_{0}$ with the property $\Sigma\left(t_{0}\right)<\infty$, then $t_{0}$ is uniquely defined (and the constant $a$ in Halász' theorem may be taken equal to $t_{0}$ ); in this case

$$
L(y)=\exp \left\{i \sum_{p \leqslant e^{y}} \frac{1}{p} \operatorname{Im}\left(f(p) p^{-i t_{0}}\right)\right\}
$$

(9) A remarkable special case of Wirsing's. Satz 1.2 is the following result: For any realvalued multiplicative function $f$, satisfying $|f| \leqslant 1$, the mean value

$$
M(f)=\prod_{p}\left\{\left(\sum_{k=0}^{\infty} p^{-k} f\left(p^{k}\right)\right)\left(1-\frac{1}{p}\right)\right\}
$$

exists.

(10) $L$ is slowly oscillating, if $L(c u) / L(u) \rightarrow 1$ for $u \rightarrow \infty$, for any real $c$ in $0<c<\infty$.

(11) This gives another solution of an old conjecture of P. Erdös. The first solution of this conjecture is contained in Wirsing [82], as mentioned before. 
There are generalizations of Halász' Theorem by several authors (see for example Levin-Timofeev [48], Tulyaganova [76], Parson-Tull [56]). (12)

\subsection{The theorem of Elliott and Daboussi}

Returning to the case of multiplicative functions with non-zero mean value, the annoying condition $|f| \leqslant 1$ in Delange's theorem was removed only in 1975 by P. D. T. A. Elliott. Define the semi-norms $\|f\|_{q}$ by

$$
\|f\|_{q}=\left\{\limsup _{x \rightarrow \infty} \frac{1}{x} \sum_{n \leqslant x}|f(n)|^{q}\right\}^{1 / q},
$$

for $q \geqslant 1$. Define a subset $\mathscr{E}_{q}$ of the set of multiplicative functions $\mathscr{M}$ as follows:

$f \in \mathscr{E}_{q}$ if and only if the following series are convergent:

$$
\begin{gathered}
S_{1}(f)=\sum_{p} \frac{1}{p}\{f(p)-1\}, \\
S_{2}^{\prime}(f)=\sum_{|f(p)| \leqslant 3 / 2} \frac{1}{p}|f(p)-1|^{2}, \\
S_{2}^{\prime \prime}(f)=\sum_{|f(p)|_{>3 / 2}} \frac{1}{p}|f(p)|^{q}, \\
S_{3}(f)=\sum_{p} \sum_{k \geqslant 2} \frac{1}{p^{k}}\left|f\left(p^{k}\right)\right|^{q} .
\end{gathered}
$$

For $q>1$ it is easy to see that $\mathscr{E}_{q}$ is a semigroup with respect to (pointwise) multiplication of functions. ${ }^{(13)}$

(12) K. H. Indlekofer [41] extended Halász' theorem in the following manner: Let $f$ be multiplicative and "uniformly summable", i.e.,

$$
\lim _{x \rightarrow \infty} \sup _{x \geqslant 1} \frac{1}{x} \sum_{n \leqslant x,|f(n)| \geqslant k}|f(n)|=0 .
$$

Assume that $M(|f|)$ exists. Then either the mean value $M(f)=0$ exists, or there exist real constants $0 \neq c_{0}$ and $a_{0}$ such that

$$
x^{-1} \sum_{n \leqslant x} f(n)=c_{0} x^{i a_{0}} \exp \left\{i A^{*}(x)\right\}+o(1) \quad \text { as } x \rightarrow \infty,
$$

where

$$
A^{*}(x)=\sum_{p \leqslant x} p^{-1} \operatorname{Im}\left\{f(p) p^{-i a_{0}}\right\} \quad \text { and } \quad \lim _{x \rightarrow \infty} \sup _{x<y<x^{2}}\left|A^{*}(y)-A^{*}(x)\right|=0 .
$$

(13) Remark. The convergence of $S_{1}(f), S_{2}^{\prime}(f)$ and $S_{2}^{\prime \prime}(f)$ is equivalent with the convergence of $S_{1}(f)$ and

$$
S_{2}^{*}(f)=\sum_{, p} p^{-1}\left\{1-|f(p)|^{q}\right\} .
$$

Thus the inessential constant $3 / 2$ is avoided. (See Heppner [34].) 
The following important result is true, giving necessary and sufficient conditions for the existence of a non-zero mean value for multiplicative functions.

TheOREM of ElliotT-Daboussi. Assume $q>1$. For any multiplicative function $f: N \rightarrow C$ the following conditions are equivalent:

$\left(\mathrm{ED}_{1}\right)\|f\|_{q}<\infty$ and the mean value $M(f)$ exists and is non-zero.

$\left(\mathrm{ED}_{2}\right) f \in \mathscr{E}_{q}$ and condition (1.8) holds.

In the case $q=2$ this theorem was proved by Elliott [17]; Daboussi and Delange [11] gave a simpler proof for the implication $\left(E D_{1}\right) \Rightarrow\left(E D_{2}\right)$.

H. Daboussi ([8], preprint) proved the theorem for $q>1$.

P. D. T. A. Elliott [21] proved the theorem independently by a different method.

W. Schwarz and J. Spilker [72] gave another proof for the implication $\left(E D_{2}\right) \Rightarrow\left(E D_{1}\right)$ by the method of $A$. Rényi [60], using the Turán-Kubilius inequality and simple ideas from functional analysis.

E. Heppner [34] deduced the same implication from a Tauberian Theorem of G. Halász [29] under weaker assumptions.

K. H. Indlekofer [38] introduced the class of "uniformly summable" functions: $f$ is called uniformly summable, if

$$
\lim _{K \rightarrow \infty}\left\{\sup _{x \geqslant 1} \frac{1}{x} \sum_{\substack{n \leqslant x \\\left|f^{n}(n)\right| \geqslant K}}|f(n)|\right\}=0 .
$$

If $f$ is uniformly summable, then $\|f\|_{1}<\infty$. Using this definition, Indlekofer's theorem reads as follows:

Let $f$ be multiplicative and $q \geqslant 1$.

(I1) If $f$ is uniformly summable with bounded semi-norm $\|f\|_{q}$, and if the mean value $M(f)$ exists and is non-zero, then $f \in \mathscr{E}_{1}$ and $f \in \mathscr{E}_{q}$ and condition (1.8) holds.

(I2) If $f \in \mathscr{E}_{1} \cap \mathscr{E}_{q}$, then $M(f)$ exists, $\|f\|_{q}<\infty$ and fis uniformly summable. Moreover $M\left(|f|^{\lambda}\right)$ exists for $\lambda=1$ and $\lambda=q$.

\subsection{Multiplicative functions of several variables}

E. Heppner ([33], [32]) showed how to deduce results concerning mean values of multiplicative functions of several variables from results on functions of one variable. For example the following result is true. ${ }^{(14)}$

Let $f: N \times N \rightarrow C$ be multiplicative. ${ }^{(15)}$ Define the functions $f_{1}, f_{2}: N$ $\rightarrow C$ by $f_{1}(n)=f(n, 1)$ and $f_{2}(n)=f(1, n)$. Let $q>1$.

(14) This result contains for example results of $H$. Delange [16].

(15) $f\left(n_{1} \cdot n_{1}^{\prime}, n_{2} \cdot n_{2}^{\prime}\right)=f\left(n_{1}, n_{2}\right) \cdot f\left(n_{1}^{\prime}, n_{2}^{\prime}\right)$, if $\left(n_{1} \cdot n_{2}, n_{1}^{\prime} \cdot n_{2}^{\prime}\right)=1$ and $f(1,1)=1$. 
(i) Assume

$$
\sum_{n \leqslant x} \sum_{m \leqslant y}|f(n, m)|^{q} \ll x y .
$$

If the mean value ${ }^{(16)} M(f)$ exists and is non-zero, and if ${ }^{(17)} \varphi_{f}\left(p, s, s^{\prime}\right) \neq 0$ in $\operatorname{Re} s \geqslant 1, \operatorname{Re} s^{\prime} \geqslant 1$ for all primes $p$, and if $\varphi_{s_{i}}(p, 1) \neq 0$ for all $p$, then the following series are convergent:

$$
\sum_{p} \frac{1}{p}\left\{f_{i}(p)-1\right\}, \quad \sum_{p} \frac{1}{p}\left\{\left|f_{l}(p)\right|^{q}-1\right\},
$$

and

$$
\sum_{p} \sum_{k \geqslant 2} p^{-k}\left|f_{i}\left(p^{k}\right)\right|^{q} \quad \text { for } i=1,2
$$

(ii) The convergence of the four series (*) (for $i=1,2)$ and of

$$
\sum_{p} \sum_{\substack{k, m \\ k+m \geqslant 2}} p^{-k-m}\left|f\left(p^{k}, p^{m}\right)\right|^{q}
$$

implies the existence of $M(f)$ and of $M\left(|f|^{q}\right)$. If $\varphi_{f}(p, 1,1) \neq 0$ for all primes $p$, then $M(f) \neq 0$.

\section{Connections with almost-periodic functions}

\subsection{Classes of almost-periodic arithmetical functions}

The theorem of Elliott-Daboussi contains more information on arithmetical functions than is expressed in it, and there are hints for this impression, for example: If $f_{1}, f_{2}$ are multiplicative, if $\left\|f_{i}\right\|_{2}<\infty$, if the mean values $M\left(f_{i}\right)$ exist and are non-zero for $i=1,2$, then the mean value $M\left(f_{1} \cdot f_{2}\right)$ exists, and under some precautions ${ }^{(18)} M\left(1 / f_{1}\right)$ also exists. ${ }^{(19)}$

In reality the conditions of Elliott's theorem characterize multiplicative functions in certain classes of almost-periodic functions. ${ }^{(20)}$

(16) $\sum_{n \leqslant x} \sum_{m \leqslant y} f(n, m) \sim M(f) x y$, if $x \rightarrow \infty$ and $y \rightarrow \infty$ independently.

(17) $\varphi_{f}\left(p, s_{1}, s_{2}\right)=\sum_{k_{1}=0}^{\infty} \sum_{k_{2}=0}^{\infty} p^{-k_{1} s-k_{2} s^{\prime}} f\left(p^{k_{1}}, p^{k_{2}}\right)$, and $\varphi_{\theta}(p, s)=\sum_{k=0}^{\infty} p^{-k s} g\left(p^{k}\right)$.

(18) Precisely: $\left|f\left(p^{k}\right)\right| \geqslant \delta$ for any prime-power $p^{k}$, where $\delta>0$. [63].

(19) See Delange [15], Schwarz [68]. For an extension to the case $M\left(f_{2}\right)=0$, see Schwarz

(20) For the special case of Delange's Theorem, dealing with multiplicative functions of modulus $|S| \leqslant 1$, see for example $H$. Daboussi and $H$. Delange [10] and W. Schwarz and J. Spilker [71], Thm. 5.1, Thm. 5.4. 
Denote by

$$
\mathscr{A}=\operatorname{Lin}_{c}\left[e_{\alpha} ; \alpha \in R / Z\right]
$$

the complex vector space of linear combinations of the exponentials $e_{\alpha}: n \mapsto e^{2 \pi i \alpha n}$, and by

$$
\mathscr{D}=\operatorname{Lin}_{c}\left[e_{a}, \alpha \in Q\right]
$$

the vector space of linear combinations of rational exponentials. Denote by

$$
\mathscr{B}=\operatorname{Lin}_{C}\left[c_{r}, r=1,2, \ldots\right]
$$

the vector space of linear combinations of Ramanujan sums

$$
c_{r}: n \mapsto \sum_{\substack{1 \leq a \leq r \\(a, r)=1}} \exp \left(2 \pi i \frac{a}{r} n\right)=\sum_{d \mid(r, n)} d \mu\left(\frac{r}{d}\right)
$$

$\mathscr{A}, \mathscr{D}$ and $\mathscr{B}$ are not only vector-spaces, but also $C$-algebras. In fact $\mathscr{B}$ is the algebra of even functions,

$$
\mathscr{B}=\{f: N \rightarrow C, \exists k: f(n)=f(\text { g.c.d. }(n, k))\} .
$$

This was proved by E. Cohen [5] elementarily and later by Schwarz and · Spilker [70] using the approximation theorem of Weierstrass-Stone. (21)

In order to enlarge these spaces, use the supremum-norm

$$
\|f\|_{u}:=\sup _{n \in \mathbb{N}}|f(n)|
$$

to get the $C$-algebra

$$
\mathscr{A}^{\mu}:=\|\cdot\|_{\mu} \text {-closure of } \mathscr{A}
$$

of uniformly-almost-periodic arithmetical functions, the algebra

$$
\mathscr{D}^{\mu}:=\|\cdot\|_{\omega} \text {-closure of } \mathscr{D}
$$

of limit periodic uniformly-almost-periodic functions, and

$$
\mathscr{B}^{\mu}:=\|\cdot\|_{\mu^{\prime}} \text {-closure of } \mathscr{B},
$$

the algebra of uniformly-almost-even functions.

The spaces just defined are very small; for example, the beautiful function $n \mapsto n^{-1} \varphi(n)$ is not in $\mathscr{A}^{\mu}$. It is possible to characterize additive (22) and multiplicative ${ }^{(23)}$ functions in $\mathscr{B}^{\mu}$ and in $\mathscr{A}^{\mu}$ :

(21) See also Knoplmacher [46], Chapter 7.

(22) See van Kampen [45], Knopfmacher [47], W. Schwarz and J. Spilker [71], § 2.

(23) See N. G. de Bruijn [4]. 
An additive function $g$ is in $\mathscr{B}^{\mu}$ iff it is in $\mathscr{A}^{\mu}$ iff $\exists \lim _{k \rightarrow \infty} g\left(p^{k}\right)$ and $\sum_{p} \sup _{k}\left|g\left(p^{k}\right)\right|<\infty$. $<\infty$.

A multiplicative function $f$ is in $\mathscr{D}^{\mu}$ iff $\exists \lim _{k \rightarrow \infty} f\left(p^{k}\right)$ and $\sum_{p} \sup _{k}\left|f\left(p^{k}\right)-1\right|$

A multiplicative function $f$ is in $\mathscr{A}^{4}$ iff there exists an integer $N$ and a Dirichlet character $\chi \bmod N$ with the properties

(i) $\lim _{k \rightarrow \infty} f\left(p^{k}\right)=0$ if $p \mid N$,

(ii) $\lim _{k \rightarrow \infty} \bar{\chi}\left(p^{k}\right) f\left(p^{k}\right) \neq 0$ exists, if $p \nmid N$,

(iii) $\sum_{p} \sup _{k}\left|\bar{\chi}\left(p^{k}\right) f\left(p^{k}\right)-1\right|<\infty$.

Hence a strongly multiplicative function is in $\mathscr{A}^{\mu}$ iff it is in $\mathscr{B}^{\mu}$. The completely multiplicative character $\chi_{4}(n)=1,0,-1,0$ for $n \equiv 1,2,3,0$ $\bmod 4$ is in $\mathscr{A}^{\mu}$, but not in $\mathscr{B}^{4}$.

Due to their smallness the spaces $\mathscr{D}^{\mu} \subset \mathscr{D}^{\mu} \subset \mathscr{A}^{\mu}$ are not very useful for number theory. With the norm $\|\cdot\|_{q}$ we get the important, larger vectorspaces

$$
\begin{aligned}
& \mathscr{A}^{q}=\|\cdot\|_{q} \text {-closure of } \mathscr{A} \text { ( } q \text {-almost-periodic-functions), } \\
& \mathscr{B}^{q}=\|\cdot\|_{q} \text {-closure of } \mathscr{B} \text { ( } q \text {-almost-even-functions). }
\end{aligned}
$$

Set-theoretic inclusions are shown in the following diagram:

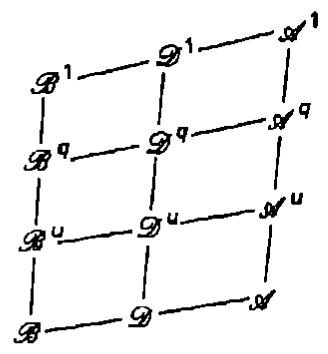

$(q \geq 1)$

There are many further set-theoretic inclusions for these spaces, which imply number-theoretic results, ${ }^{(24)}$ for example:

$$
\begin{gathered}
\mathscr{A}^{\mu} \cdot \mathscr{A}^{q} \subset \mathscr{A}^{q}, \quad \mathscr{B}^{\mu} \cdot \mathscr{B}^{q} \subset \mathscr{B}^{q}, \\
\mathscr{A}^{q} \cdot \mathscr{A}^{\prime} \subset \mathscr{A}^{1}, \quad \mathscr{B}^{q} \cdot \mathscr{B}^{q^{\prime}} \subset \mathscr{B}^{1}, \quad \text { if } \quad 1 / q+1 / q^{\prime}=1 .
\end{gathered}
$$

Relation (2.3) follows from Hölder's inequality

$$
\|f\|_{1} \leqslant\|f\|_{q} \cdot\|f\|_{q^{\prime}}, \quad \text { if } \quad 1 / q+1 / q^{\prime}=1 .
$$

(24) See Section 3. For further results see Lemmas 5.2, 5.3, 5.4. 
Furthermore, if $f \in \mathscr{A}^{q}$ [resp. in $\left.\mathscr{A}^{q}\right]$ then $|f|, \operatorname{Re} f, \operatorname{Im} f, \bar{f}$ are in $\mathscr{A}^{q}$ [resp. in $\left.\mathscr{B}^{2}\right]$.

If $f \in \mathscr{A}^{1}$, then $M(f)$ exists, and the Fourier-coefficients $\hat{f}(\alpha)$ and Ramanujan-coefficients $a_{r}(f)$ exist. If $f \in \mathscr{A}^{1}$, then the shifted functions

$$
f_{(a)}: n \mapsto f(a+n) \quad(a \in Z)
$$

and the pointwise products $g \cdot f$ for any periodic function $g$ (or for any uniformly-almost-periodic g) are in $\mathscr{A}^{1}$. If $f_{1}, \ldots, f_{k} \in \mathscr{A}^{k}$, then $F \in \mathscr{A}^{1}$, where

$$
F(n)=\prod_{x=1}^{k} f_{x}\left(a_{x} n+b_{x}\right)
$$

with integers $a_{x}>0, b_{x}$.

The structural property of "almost-evenness" for $\mathscr{B}^{4}$ is very useful. By a simple approximation argument ${ }^{(25)}$ this property implies:

If $f \in \mathscr{B}^{1}$ is real-valued, then $e^{i t f}: n \mapsto e^{i t f(n)}$ is in $\mathscr{B}^{1}$ for any real $t$.

Due to the orthogonality relations

$$
M\left(c_{r} c_{r^{\prime}}\right)= \begin{cases}0, & \text { if } r \neq r^{\prime} \\ \varphi(r), & \text { if } r=r^{\prime}\end{cases}
$$

elements of linear algebra give

Bessel's ineQuality. If $f \in \mathscr{B}^{2}$, then

$$
\sum_{r}\left|a_{r}(f)\right|^{2} \varphi(r) \leqslant\|f\|_{2}^{2}
$$

where $a_{r}(f)=\frac{1}{\varphi(r)} M\left(f c_{r}\right)$.

For an application in the next section we shall need

Parseval's equality. If $f \in \mathscr{B}^{2}$, then

$$
\sum_{r=1}^{\infty}\left|a_{r}(f)\right|^{2} \varphi(r)=\|f\|_{2}^{2}
$$

The proof can be based on an isomorphism $\mathscr{B}^{2} / \mathscr{N}^{2} \cong L^{2}\left(N^{*}, \mu\right)$ of $\mathscr{B}^{2}$ modulo null-functions ${ }^{(26)}$ to an $L^{2}$-space over a compactification $N^{*}$ of $N$ with a suitable measure $\mu$. For details see Knopfmacher [47].

(25) If $g=\sum a_{r} c_{r}$ is a finite linear combination of Ramanujan sums, then $e^{\text {itg }}$ is even, and hence again in $\operatorname{Lin}_{c}\left[c_{r}\right]$. This type of argument does not seem to be available for $\mathscr{A}^{1}$.

(26) $\mathscr{N}^{2}=\left\{f \in \mathscr{O}^{2},\|f\|_{2}=0\right\}$. 


\section{Applications to number theory and to power-series}

\subsection{Number-theoretical applications}

For functions $f$ in $\mathscr{C}^{1}$ the mean value exists; using inclusion relations between the spaces defined in Section 2, we obtain many results on the existence of mean values for arithmetical functions. ${ }^{(27)}$

(1) If $f \in \mathscr{A}^{1}$, then the mean values $M(f), M(\operatorname{Re} f), M(\operatorname{Im} f), M(|f|)$ and $M(f \cdot g)$ exist for any $g \in \mathscr{A}^{\mu}$ (for example for Dirichlet characters $g$ or for characteristic functions of residue classes).

(2) If $f \in \mathscr{A}^{1}$ then the Fourier-coefficients $\hat{f}(\alpha)=M\left(f \bar{e}_{\alpha}\right)$ and $a_{r}(f)$ $=\frac{1}{\varphi(r)} M\left(f c_{r}\right)$ exist.

(3) If $f, g \in \mathscr{A}^{2}$, then $M\left(f_{(a)}\right)$ exists, where $g_{(a)}(n)=g(a+n)$. If $f \in \mathscr{A}^{q}$, $g \in . \mathscr{d}^{q^{\prime}}$ (where $1 / q+1 / q^{\prime}=1$ ), then $M\left(f_{(a)} g_{(b)}\right)$ exists. ${ }^{(28)}$

More generally:

(4) Given functions $f_{1}, \ldots, f_{k}$ in $\mathscr{A}^{k}$ and integers $a_{x}>0, b_{x}, c_{x}>0(x$ $=1, \ldots, k ; k$ an integer $)$, then

$$
\lim _{x \rightarrow \infty} \frac{1}{x} \sum_{n \leqslant x} \prod_{x=1}^{k} f_{x}\left(\frac{a_{x} n+b_{x}}{c_{x}}\right)
$$

exists, where $f_{x}(y)$ is defined to be zero, if $y \notin N$.

(This generalizes results of L. Lucht [50], [51], if the results of Section 4 are used, giving sufficient conditions for $f \in \mathscr{A}^{k}$ in the case of multiplicative or additive functions.)

(5) If $f \in \mathscr{A}^{q}$ for any $q \geqslant 1$, if the image $f(N)$ is contained in an open set $U \subset C$ with compact closure $\bar{U}$, and if $\psi: U \rightarrow C$ is continuous, then the composed function $\psi$ of is in $\mathscr{A}^{q}$ for any $q \geqslant 1$.

Examples are $1 / f, \exp f, \log f, \cos f$, etc. The proof is by a standard application of the Weierstrass approximation theorem.

(6) If (29) $f \in \mathscr{B}^{1}$, if $f(N) \subset U$ and if $\psi: U \rightarrow C$ is Lipschitzcontinuous, ${ }^{(30)}$ then $\psi$ of is in $\mathscr{B}^{1}$.

A very important example is $\psi(x)=e^{i t x}$ for real $t$.

COROLLARY. If $g$ is a real-valued arithmetical function in $\mathscr{B}^{1}$, then there is a limit-distribution for $g$, that means

$$
\lim _{N \rightarrow \infty} \frac{1}{N} \#\{n \leqslant N ; g(n) \leqslant x\}=\Psi_{\theta}(x)
$$

(27) regardless of being multiplicative or additive.

(28) Example: The function $\mu^{2}$ is in for any $q>1$ (see Section 4 ), hence $M\left(\mu^{2} \cdot f_{(a)}\right)$ exists for any $f \in . r /$, where $q>1$. This result is sharpened in Section 5, Lemma 5.3.

(29) Warning: The prool does not apply to $f \in \mathscr{d}^{1}$.

(30) i.e, $\left|\psi(x)-\psi\left(x^{\prime}\right)\right| \leqslant K\left|x-x^{\prime}\right|$ for some constant $K$ and for $x, x^{\prime} \in U$. 
exists in the sense of weak convergence, i.e. relation (3.1) is true for any point of continuity of $\Psi_{g}$.

In order to prove this, according to the continuity theorem for characteristic functions (Lukacs [54], Thm. 3.6.1) it is sufficient to prove the existence of the mean values

$$
M_{t}=M(n \mapsto \exp \{i \operatorname{tg}(n)\})
$$

for real $t$ (this follows from $e^{\mathrm{i} t \theta} \in \mathscr{B}^{1}$ ) and the continuity of $t \mapsto M_{t}$ at $t=0$; this follows from the estimate

$$
\left|\lim _{N \rightarrow \infty} \frac{1}{N} \sum_{n \leqslant N}\left(e^{i t \theta(n)}-1\right)\right| \leqslant \varlimsup_{N \rightarrow \infty} \frac{1}{N} \sum_{n \leqslant N}|t g(n)|=|t| \cdot\|g\|_{1} .
$$

\subsection{Power-series with multiplicative coefficients}

Let us recall the theorems of G. Pólya [57], [58] and G. Szegö [75]: If the coefficients $f^{\prime}(n)$ of a power series

$$
F(z)=\sum_{n=1}^{\infty} f(n) z^{n}
$$

with radius of convergence equal to 1 are integers [resp. assume only finitely many values], then either $F(z)$ represents a rational function or it is noncontinuable beyond the unit circle.

It suggests itself to ask, ${ }^{(31)}$ what will happen, if the coefficients $f(n)$ of $F(z)$ are values of a multiplicative function $f$. Will the same answer be true?

L. Lucht and F. Tuttas [53] and L. Lucht [52] answered this question in the affirmative: If $f$ is multiplicative with finite norm $\|f\|_{2}$, and if the mean value $M(f)$ exists and is non-zero, then the power series $F(z)$ is noncontinuable if and only if

$$
\frac{f\left(p^{k-1}\right)}{\varphi\left(p^{k}\right)} \neq \sum_{v=k}^{\infty} \frac{f\left(p^{v}\right)}{p^{v}}
$$

for infinitely many prime powers $p^{k}$; otherwise $F(z)$ represents a rational function. (32)

For example the power series

$$
\sum_{1}^{\infty} \frac{\varphi(n)}{n} z^{n}
$$

is non-continuable beyond the unit circle.

(31) The author posed this question at the number theory conference in Oberwolfach 1978.

(32) Lucht [52] contains more general results. 
However, inspite of this nice result, the posed question is not the right question. The property of being multiplicative only plays a very subordinate rôle. The following result is true.

Let $f \in \mathscr{B}^{2}$. Then

(a) $F(z)$ is non-continuable, if infinitely many Ramanujan coefficients $a_{r}(f)$ $=M\left(f c_{r}\right) / \varphi(r)$ are non-zero.

(b) If only finitely many $a_{r}(f)$ are non-zero, and if $f(n)=\sum a_{r} c_{r}(n)$, i.e. if $f$ is represented by its Ramanujan expansion, ${ }^{(33)}$ then $F(z)$ is rational.

Remark 1. If $f$ is in $\mathscr{B}^{2}$, if $f$ is multiplicative or additive, and if $M(f) \neq 0$ in the multiplicative case, then the Ramanujan expansion

$$
f(n)=\sum_{r=1}^{\infty} a_{r} c_{r}(n)
$$

is pointwise convergent. ${ }^{(34)}$ Thus the condition $f=\sum a_{r} c_{r}$ in the case (b) of the theorem may be dropped if $f$ is multiplicative or additive.

Using explicit formulae for the Ramanujan coefficients $a_{r}(f)$, in both cases ( $f$ multiplicative or $f$ additive), the condition " $a_{r}(f) \neq 0$ infinitely often" is equivalent with Lucht's condition (3.3).

Remark 2. The assumption $f \in \mathscr{B}^{2}$ may be replaced by $f \in \mathscr{B}^{1}$.

For the proof, Parseval's equation has to be replaced by a result of A. Hildebrand

$$
\lim _{K \rightarrow \infty}\left\|f-\sum_{r \mid K !} a_{r}(f) c_{r}\right\|_{1}=0 .
$$

The important feature of (3.4) is that the coefficients of the even function $\sum_{r \mid K !} a_{r}(f) c_{r}$ approximating $f$ in $\|\cdot\|_{1}$ are not changed, when $K$ is increased.

Remark 3. An extension of the result is possible, since differentiation does not destroy the property of being rational or non-continuable. Hence the assumption $f \in \mathscr{B}^{2}$ may be replaced by $n^{-k} f(n) \in \mathscr{B}^{2}$ for some integer $k$ $\geqslant 0$.

Remark 4. The result is no longer true, if $f \in \mathscr{B}^{2}$ is replaced by $f \in \mathscr{A}^{4}$ or by $f \in \mathscr{A}^{\mu}$. For example, the function $f$, defined by the uniformly convergent series

$$
f(n)=\sum_{k=1}^{\infty} \frac{1}{k^{2}} e^{2 \pi i(1 / k) n}
$$

(33) Thus $f \in \mathscr{D}$ in case (b).

(34) For multiplicative functions: Schwarz [65], [66], Tuttas [77], Warlimont [78]. For additive functions see for example Hildebrand-Spilker [37]. 
is in $\mathscr{A}^{\mu}$, but

$$
\sum_{n=0}^{\infty} f(n) z^{n}=\sum_{k=1}^{\infty} \frac{1}{k^{2}} \cdot \frac{1}{1-e^{(2 \pi i \cdot(1 / k))} \cdot z}
$$

is continuable beyond $|z|=1$ and not rational.

Idea of proof. Since $f \in \mathscr{B}^{2}$, the function $f$ may be approximated in $\|\cdot\|_{2}$ by the beginning of its Ramanujan expansion

$$
\left\|f-\sum_{r=1}^{R} a_{r}(f)^{2} c_{r}\right\|_{2}=\sum_{r>R}\left|a_{r}(f)\right|^{2} \varphi(r)<\varepsilon,
$$

if $R>R_{0}(\varepsilon)$ is sufficiently large.

The function

$$
\mathscr{R}(z)=\sum_{n=1}^{\infty}\left\{\sum_{r=1}^{R} a_{r}(f) c_{r}(n)\right\} z^{n}
$$

is rational, by a simple calculation we obtain

$$
\mathscr{R}(z)=\sum_{r=1}^{R} a_{r}(f) \sum_{\omega \bmod r} \frac{\omega z}{1-\omega z}
$$

where $\omega$ runs through the primitive $r$ th roots of unity

$$
\omega_{a, r}=\exp \left(2 \pi i \frac{a}{r}\right), \quad(a, r)=1
$$

Using $\|\cdot\|_{1} \leqslant\|\cdot\|_{2}$, by partial summation ${ }^{(35)}$ the estimate (3.5) implies

$$
\left|\sum_{1}^{\infty} f(n) z^{n}-\mathscr{R}(z)\right|<\frac{2 \varepsilon}{1-|z|},
$$

if $|z|$ is near 1 . Hence

$$
\left|\sum_{1}^{\infty} f(n) z^{n}\right| \rightarrow \infty
$$

if $z \rightarrow \omega_{a, r}$ along the straight line $z=t \omega_{a, r}, 0<t<1$, and if $a_{r}(f) \neq 0$.

If $a_{r}(f) \neq 0$ infinitely often, then the singularities $\omega_{a, r},(a, r)=1$, are dense on $|z|=1$, and the result concerning the non-continuability of $\sum f(n) z^{n}$ is proved.

(35) The partial summation only needs an approximation of $f$ in $\|\cdot\|_{1}$. 


\subsection{Some power series bounded on the negative real axis}

L. Rubel and K. Stolarsky [61] posed the following problem: Determine all subsets $N_{1}$ of the set $\boldsymbol{N}$ of natural numbers for which the power series

$$
\sum_{n \in N_{1}} \frac{1}{n !} x^{n}
$$

shares with the exponential function the property of being bounded on the negative real axis.

Their solution of this problem shows that there are only a few possibilities for $N_{1}$, just the following six subsets $N_{1} \subset N$ have the desired property:

$$
\begin{array}{ll}
N_{1}=\emptyset, \quad N_{1}=N, & \\
N_{1}=\{n, n \equiv 0,1 \bmod 4\}, & N_{1}=\{n, n \equiv 2,3 \bmod 4\}, \\
N_{1}=\{n, n \equiv 0,3 \bmod 4\}, & N_{1}=\{n, n \equiv 1,2 \bmod 4\} .
\end{array}
$$

The corresponding functions are

$$
0, \quad e^{x}-1, \quad \frac{1}{2}\left(e^{x}+\cos x+\sin x\right)-1,
$$

$\frac{1}{2}\left(e^{x}-\cos x-\sin x\right), \quad \frac{1}{2}\left(e^{x}+\cos x-\sin x\right)-1, \quad \frac{1}{2}\left(e^{x}-\cos x+\sin x\right)$.

In analogy we pose the problem of determining all multiplicative or additive functions $f \in \mathscr{B}^{2}$ (with $M(f) \neq 0$ in the multiplicative case), for which the function

$$
E_{f}(z)=\sum_{n=1}^{\infty} \frac{f(n)}{n !} z^{n}
$$

is bounded on the negative real axis.

The method of proof is similar to that of Rubel and Stolarsky. The solutions are

(a) in the multiplicative case: $f=1$ or $f$ is periodic modulo 4,

$$
f(n)=\left\{\begin{array}{lll}
1 & \text { if } & n \equiv 1,3 \bmod 4 \\
1-c & \text { if } & n \equiv 2 \bmod 4 \\
1+c & \text { if } & n \equiv 0 \bmod 4
\end{array}\right.
$$

The corresponding functions are $e^{x}-1$ and $e^{x}-1-c(\cos x-1)$ (with an arbitrary parameter $c$ ).

(b) in the additive case:

$$
\begin{gathered}
g(n)=\left\{\begin{array}{lll}
c & \text { if } & 4 \mid n, \\
-c & \text { if } & 2 \| n, \\
0 & \text { if } & 2 \nmid n,
\end{array}\right. \\
E_{\theta}(z)=c(\cos z-1)
\end{gathered}
$$


Sketch of proof. Consider the Laplace transform ${ }^{(36)}$

$$
\mathscr{L}_{f}(z)=\int_{0}^{\infty} E_{f}(-t) e^{-t z} d t=\sum_{n=1}^{\infty}(-1)^{n} f(n) \frac{1}{z^{n+1}} .
$$

The first representation shows that $\mathscr{L}_{j}(z)$ is holomorphic in $\operatorname{Re} z>0$, since $E_{f}(-t)$ is bounded for $t>0$, and the second representation implies that $\mathscr{L}_{f}(z)$ is holomorphic in $|z|>1$, since $\|f\|_{2}<\infty$ easily gives $|f(n)| \leqslant C \sqrt{n}$. Hence

$$
-\mathscr{L}_{f}(-1 / z)=z \sum_{n=1}^{\infty} f(n) z^{n}
$$

is continuable beyond the unit circle; according to Section $3.2 \mathscr{L}_{f}(-1 / z)$ is rational and $f \in \mathscr{B}$, thus

$$
f(n)=\sum_{r=1}^{R} a_{r} c_{r}(n)
$$

Inserting (3.7) into (3.6) we obtain

$$
E_{f}(z)=\sum_{r=1}^{R} a_{r} \sum_{\substack{1 \leqslant a \leqslant r \\(a, r)=1}}\left(e^{\omega_{a, r^{z}}}-1\right)
$$

and this function is an exponential polynomial. Boundedness on $x<0$ implies $a_{r}=0$ for any exponent $\omega_{a, r}=\exp \left(2 \pi i \frac{a}{r}\right)$ with $\operatorname{Re}\left(\omega_{a, r}\right)<0$. For $r \neq 1,4,6$ there are primitive roots of unity $\omega$ with $\operatorname{Re} \omega<0$, and so $a_{r}=0$ unless $r=1,4,6$.

In the multiplicative case the function $r \mapsto \frac{1}{M(f)} a_{r}$ is multiplicative; therefore $a_{6}=0$, since $a_{2}=0$, and

$$
1=f(1)=a_{1} c_{1}(1)+a_{4} c_{4}(1)=a_{1},
$$

and so

$$
f(n)=1+a_{4} c_{4}(n)
$$

gives all the solutions of our problem.

In the additive case $a_{r}=0$, if $r$ is not a power of a prime, hence $a_{6}=0$. Moreover,

$$
0=f(1)=a_{1} c_{1}(1)+a_{4} c_{4}(1)=a_{1}
$$

(36) "Borel-transform" in the theory of entire functions. 
and thus $a_{1}=0$, and we obtain the solutions

$$
f(n)=a_{4} c_{4}(n) \text {. }
$$

Remark. Th. Maxsein dealt with the case of additive and multiplicative functions $f$, with the property that the estimate

$$
\left|\sum_{n=1}^{\infty} \frac{1}{n !} f(n) z^{n}\right| \leqslant c \cdot e^{\theta|z|}
$$

holds on the negative real axis, where $0 \leqslant \theta<1$. There are no new solutions as long as $\theta<1 / 2$. For $\theta=1 / 2$

$$
a_{1}\left(e^{z}-1\right)+\left(a_{1}-1\right)\left(e^{Q z}-1+e^{\bar{a} z}-1\right)
$$

where $\varrho=-\frac{1}{2}+\frac{1}{2} i \sqrt{3}$, is a new solution. The number of solutions increases as $\theta \rightarrow 1-$.

\section{Additive and multiplicative functions in $20^{9}$}

It seems to be very difficult to characterize arithmetical functions in $\mathscr{A}^{g}$ or $\mathscr{g}^{9}$ by well applicable conditions. However, it is possible to do this for additive and for multiplicative functions. In this way one gets lots of functions for which the results of Section 3 may be applied.

\subsection{Additive functions}

The first result characterizes additive functions $g: N \rightarrow C$ with finite norm $\|g\|_{q}, q \geqslant 1$.

Suppose $q \geqslant 1$ and $g$ additive. Then $\|g\|_{q}<\infty$ if and only if

$$
\begin{gathered}
\sup _{N \in \mathbb{N}}\left|\sum_{\substack{p \leqslant N \\
|g(p)| \leqslant 1}} \frac{g(p)}{p}\right|<\infty, \\
\sum_{|g(p)| \leqslant 1} \frac{1}{p}|g(p)|^{2}<\infty,
\end{gathered}
$$

and

$$
\sum_{\substack{p \\\left|g\left(p^{k}\right)\right|>1}} \sum_{k \geqslant 1} \frac{1}{p^{k}}\left|g\left(p^{k}\right)\right|^{q}<\infty .
$$

The characterization of additive functions in $\mathscr{B}^{q}$ is as follows.

Suppose $q \geqslant 1$ and $g$ additive. Then the following conditions are equivalent: 
$\left(A_{1}\right) g \in 10^{9}$.

$\left(\mathrm{A}_{2}\right)$ The mean value $M(g)$ exists and $\|g\|_{q}<\infty$.

$\left(\mathrm{A}_{3}\right)$ The series

$$
\sum_{|g(p)| \leqslant 1} \frac{1}{p} g(p)
$$

and the series (4.1.2) and (4.1.3) are convergent.

Addendum. For additive functions $g \in \mathscr{B}^{q}(q \geqslant 1)$ the Ramanujan coeffcients are given by

$$
\begin{gathered}
a_{1}=M(g)=\sum_{p}\left(1-\frac{1}{p}\right) \sum_{k=1}^{\infty} \frac{g\left(p^{k}\right)}{p^{k}}, \\
a_{r}= \begin{cases}-\frac{g\left(p^{k-1}\right)}{p^{k}}+\left(1-\frac{1}{p}\right) \sum_{m \geqslant k} \frac{g\left(p^{m}\right)}{p^{m}}, & \text { if } r=p^{k}, \\
0, \quad \text { if } r \geqslant 2 \text { is not a power of a prime. }\end{cases}
\end{gathered}
$$

The Ramanujan expansion

$$
\sum_{r=1}^{\infty} a_{r}(g) c_{r}(n)=g(n)
$$

is pointwise convergent.

These results are proved in Hildebrand and Spilker [37]. Another proof of the first result using a generalized form of the Turán-Kubilius inequality, is given in Elliott [22], Theorem 4. We do not reproduce these proofs here. K. H. Indlekofer [Preprint Oberwolfach, Nov. 1980] announced (without proofs) the equivalence of $\|g\|_{q}<\infty$ and $\{(4.1 .1),(4.1 .2)\}$ even in the case $q>0$. (37)

Moreover, Indlekofer [43] announced that for $q \geqslant 1$ and for real-valued additive $g$ the existence of a limit distribution $\Psi_{\theta}$ for $g$ and the relation $\int_{-\infty}^{\infty}|y|^{q} d \Psi_{\theta}(y)<\infty$ are equivalent with the convergence of the series (4.2), (4.1.2) and (4.1.3).

Of course this follows from the equivalence $\left(A_{1}\right) \Leftrightarrow\left(A_{3}\right)$ and Section 3.1 (6).

Remark 1. Hartmann and Wintner [31] proved that $\left(\mathrm{A}_{3}\right)$ is equivalent with $g \in \mathscr{A}^{q}$ (in the cases $q=1$ or $q=2$, and they stated the result for any $q$ $\geqslant 1$, p. 758). Hence, denoting the set of additive functions by Add, the

(37) For $0<q<1$ the definition of $\|f\|_{q}$ is slightly different from that given in Section 2 . 
following relation is true

$$
. g^{q} \cap \text { Add }=\mathscr{B}^{q} \cap \text { Add } \quad(q>1) .
$$

Remark 2. There are connections with a charming problem of P. Erdös [23]. This problem is to characterize the logarithm as an additive function with some additional properties, for example: An additive function $g$ satisfying

$$
\begin{aligned}
& g(n+1)-g(n) \rightarrow 0 \quad \text { as } \quad n \rightarrow \infty \\
& {\left[\text { resp. } \sum_{n \leqslant x}|g(n+1)-g(n)|=o(x)\right]}
\end{aligned}
$$

is of the form $g=c \cdot \log$ (see Erdös [23], resp. Wirsing [83]).

We do not treat this topic in this survey and mention the papers of Rényi [59], Elliott [19], Wirsing [85] and the Literature quoted there. (38)

\subsection{Multiplicative functions with mean value $M(f) \neq 0$}

In his preprint [6] $\mathrm{H}$. Daboussi proves the following theorem:

Suppose $q>1$ and $f$ multiplicative. If then ${ }^{(39)}$

$\left(\mathrm{ED}_{1}\right)\|f\|_{q}<\infty$ and the mean value $M(f)$ exists and is non-zero,

$\left(\mathrm{ED}_{2}\right) f \in \mathscr{E}_{q}$ and condition (1.8) holds.

On the other hand, $\left(\mathrm{ED}_{2}\right)$ implies that $f$ is in $\mathscr{A}^{9}$ and is limit-periodic ${ }^{(40)}$ hence $f \in \mathcal{D}^{q}$.

Daboussi's proof uses Dirichlet series and techniques of Halász. A result like Daboussi's theorem ought to be proven by functional-analytic means, without using too heavy tools from number theory, and one of the aims of this survey is to sketch a proof of the following slightly different result, using simple ideas from functional analysis and the theory of almost-periodic functions.

Suppose $f$ is multiplicative.

$\left(\mathrm{M}_{1}\right)$ If $q \geqslant 1$ and $f \in \mathscr{E}_{q}$, then $f \in \mathscr{B}^{q}$.

Addendum: If (1.8) holds, then $M(f) \neq 0$.

$\left(\mathrm{M}_{2}\right)$ If $q \geqslant 1$ and $f \in \mathscr{d}^{q}$ with mean value $M(f) \neq 0$, then $f \in \mathscr{E}_{q}$ and (1.8) holds.

(38) Wirsing's (best-possible) result (see Wirsing [85] is: If $g$ is a completely additive function, satisfying

$$
g(n)-g(n+1)=o(\log n) \quad \text { as } \quad n \rightarrow \infty,
$$

then $g$ is a constant multiple of the logarithm, $g=c \cdot \log$.

(39) Recall that $f \in \delta_{q}$ was defined at the beginning of Section 1.4 by the convergence of the series $S_{1}, S_{2}^{\prime}, S_{2}^{\prime \prime}$ and $S_{3}$. Condition (1.8) means $\sum_{k=0}^{\infty} p^{-k} f\left(p^{k}\right) \neq 0$ for any prime $p$.

${ }^{(40)} f$ is limit-periodic, if the Fourier-coefficients $f(\alpha)$ vanish for irrational numbers $\alpha$. 
Remark. It would be highly desirable to replace the assumption $f \in \mathscr{A}^{q}$ by the existence of $M(f) \neq 0$ and $\|f\|_{q}<\infty$. However, in our proof, it would be necessary to know about the existence of $M\left(|f|^{q / 2}\right)$, and it seems that this could be deduced only from Halász-like theorems.

H. Daboussi [8], went further and gave a characterization of multiplicative functions in $\mathscr{A}^{q}$ with a non-void Fourier-Bohr spectrum. Define the spectrum of an arithmetical function as

$$
\operatorname{spec}(f)=\left\{\alpha \in R / Z ; \limsup _{x \rightarrow \infty} \frac{1}{x}\left|\sum_{n \leqslant x} f(n) e^{-2 \pi i n x}\right|>0\right\},
$$

and so, for functions $f$ in $\mathscr{A}^{1}$,

$$
\operatorname{spec}(f)=\{\alpha \in \boldsymbol{R} / Z ; \hat{f}(\alpha) \neq 0\} \text {. }
$$

Then Daboussi's "Théorème principal" is as follows:

Assume $q \geqslant 1$ and $f$ multiplicative. Then $f$ is in $\mathscr{A}^{4}$ with a non-void Fourier-Bohr spectrum if and only if there exists a Dirichlet character $\chi$ with the property that the four series

$$
\begin{gathered}
\sum_{p} p^{-1}\{\chi(p) f(p)-1\}, \\
\sum_{|f(p)| \leqslant 2} p^{-1}|\chi(p) f(p)-1|^{2}, \\
\sum_{p,|f(p)|>2} p^{-1}|f(p)|^{q},
\end{gathered}
$$

and

$$
S_{3}(f)=\sum_{p} \sum_{k \geqslant 2} p^{-k}\left|f\left(p^{k}\right)\right|^{q}
$$

are convergent.

If these conditions are satisfied, then $f$ is limit-periodic (i.e. $f \in \mathscr{D}^{q}$ resp. $\operatorname{spec}(f) \subset Q$ ).

Daboussi's Theorem 2 characterizes positive multiplicative functions in $\mathscr{A}^{9}$ with non-void spectrum by the conditions (4.6.1)-(4.6.4), where now the Dirichlet character $\chi$ is equal to the constant function 1 .

K. H. Indlekofer [42] ${ }^{(41)}$ extends this theorem:

Assume $\alpha \geqslant 1$. Then $f \in \mathscr{A}^{a}$ with $\operatorname{spec}(f) \neq \emptyset$ is equivalent with the convergence of the four series mentioned above for all $q$ in $1 \leqslant q \leqslant \alpha$ and is equivalent with

(4.7) $\|f\|_{a}<\infty, f$ uniformly summable and there exists a Dirichlet character $\chi$ such that $M(f \chi)$ exists and is different from zero.

(41) Corollary 1; see also Indlekoler [40]. 


\section{Tooks from number theory and functional analysis}

\subsection{The relationship theorem}

Multiplicative functions are determined by their values at the primepowers. Therefore we may expect that multiplicative functions have "similar" properties, if they do not differ "too much" on the primes. For example, if one function has a mean value or Fourier coefficients, then the other one ought to have a mean value or Fourier coefficients too. This often used idea allows to reduce proofs to simpler special cases by deleting a thin set of "bad primes" or "bad prime-powers". If one has some control on the values at prime-powers one may replace a multiplicative function by a strongly or completely multiplicative function "without loss of generality".

The following result is due to E. Heppner and the author [35], weaker forms were proved before by several authors (for example L. Lucht [49], W. Schwarz [64] or H. Delange [12]). if

Define two multiplicative functions $f, g$ to be related, abbreviated $f \sim g$,

$$
\sum \frac{1}{p}|f(p)-g(p)|<\infty
$$

Denote by $\mathscr{G}$ the set of functions

$$
\mathscr{G}=\left\{f: N \rightarrow C, f \text { multiplicative, } \sum_{p} \sum_{k \geqslant 2} p^{-k}\left|f\left(p^{k}\right)\right|<\infty, \sum_{\dot{p}}\left|\frac{f(p)}{p}\right|^{2}<\infty\right\},
$$

and by $\mathscr{G} *$ its subset

$$
\mathscr{G}^{*}=\left\{f \in \mathscr{G} ; \varphi_{f}(p, s):=1+\frac{f(p)}{p^{s}}+\frac{f\left(p^{2}\right)}{p^{2 s}}+\ldots \neq 0 \text { in } \operatorname{Re} s \geqslant 1\right.
$$

Then the following result is true. for any prime $p\}$.

Lemma 5.1. Let $f, g$ be multiplicative and related, $f \sim g$; assume

$$
f, g \in \mathscr{G} \text { and } f \in \mathscr{G}^{*} \text {. }
$$

Then there exists a (multiplicative) function $h$, satisfying

$$
g=f * h \quad \text { and } \quad \sum_{n=1}^{\infty}\left|\frac{h(n)}{n}\right|<\infty .
$$

Remark 1. This lemma was extended to multiplicative functions of several variables by E. Heppner [32]. 
Remark 2. $\mathscr{G}$ and $\mathscr{G}^{*}$ are closed ${ }^{(42)}$ with respect to Dirichlet convolution *, defined by

$$
f * g: n \mapsto \sum_{d \mid n} f(d) * g\left(\frac{n}{d}\right),
$$

and $f \in \mathscr{G}^{*}$ implies $\bar{f} \in \mathscr{G}^{*}$, where $\bar{f}$ is the convolution inverse of $f(f * \bar{f}=\varepsilon)$.

Remark 3. If the condition $\varphi_{f}(p, s) \neq 0$ in $\operatorname{Re} s \geqslant 1$ is violated for some primes, the conclusion of Lemma 5.1 remains true, if the condition

$$
f\left(p^{k}\right)=g\left(p^{k}\right) \quad \text { for } k=1,2, \ldots
$$

is assumed for these "bad" primes.

The importance of Lemma 5.1 is due to the following:

COROLlary. Let $f, g$ satisfy the assumptions of Lemma 5.1. Then:

(1) If $M(f)$ exists, then $M(g)$ exists ${ }^{(43)}$ and

$$
M(g)=M(f) \sum_{1}^{\infty} \frac{h(n)}{n}=M(f) \prod_{p}\left(1+\frac{h(p)}{p}+\frac{h\left(p^{2}\right)}{p^{2}}+\ldots\right) .
$$

(2) If $f \in \mathscr{B}^{1}$, then $g \in \mathscr{B}^{1}$.

(3) If $f \in \mathscr{A}^{1}$, then $g \in \mathscr{A}^{1}$.

(4) If $\sum \frac{1}{n} f(n)$ is convergent, then the series $\sum \frac{1}{n} g(n)$ is convergent too.

(5) If all the Fourier coefficients $\hat{f}(\alpha)$ exist, then all the $\hat{g}(\alpha)$ exist.

For the proof of Lemma 5.1 one describes the relation $h=g * f$ with the aid of Dirichlet series.

$$
\sum \frac{h(n)}{n^{s}}=\sum \frac{g(n)}{n^{s}} / \sum \frac{f(n)}{n^{s}}=\left\{\sum \frac{g(n)}{n^{s}} \zeta^{-1}(s)\right\}\left\{\sum \frac{f(n)}{n^{s}} \zeta^{-1}(s)\right\}^{-1} .
$$

The main difficulty is then to show that the inverse of an absolutely convergent Dirichlet series is again absolutely convergent, and this is achieved by a theorem of Wiener's type due to Hewitt and Williamson. ${ }^{(44)}$

$$
\begin{aligned}
& \text { If } \sum_{1}^{\infty}\left|a_{n}\right|<\infty \text {, then } \\
& \qquad\left|\sum_{1}^{\infty}\left(a_{n} / n^{s}\right)\right| \geqslant \delta>0 \text { in } \operatorname{Re} s \geqslant 0
\end{aligned}
$$

(42) $f, g \in \mathscr{G}$ (resp. $\in \mathscr{G}^{*}$ ) implies $f * g \in \mathscr{G}$ (resp. $\in \mathscr{G}^{*}$ ).

(43) See also A. Wintner [80].

(44) In his generalization of Lemma 5.1 E. Heppner [32] replaces this result by simpler results from the theory of Banach algebras. 
is equivalent to

$$
\sum_{1}^{\infty}\left|b_{n}\right|<\infty
$$

where the sequence $b_{n}$ is defined by

$$
\left(\sum a_{n} n^{-s}\right)\left(\sum b_{n} n^{-s}\right)=1
$$

The proof of this result uses Gelfand's theory of commutative Banach algebras. A proof by complex function theory was given by A. E. Ingham [44], and an elementary proof, modelled after D. J. Newman [55] was given by J. Spilker and W. Schwarz [74].

\subsection{Lemmata concerning spaces of almost-periodic functions}

LEMMA 5.2. If $q \geqslant 1, g \in \mathscr{B}^{q}$ and $g \geqslant 0$, then $g^{q / 2} \in \mathscr{B}^{2}$.

The same result is true for $\mathscr{A}^{q}$ resp. for $\mathscr{D}^{q}$.

This is a simple result from the theory of almost-periodic functions; a proof is given in the appendix of Daboussi's paper [8]: ${ }^{(45)}$ Use the Weierstrass approximation theorem to replace $\{\sup (0, u)\}^{1 / 2 q}$ by a polynomial $Q(u)$ and use that $w \in \mathscr{B}$ implies $Q \circ w \in \mathscr{B}$.

LEMMA 5.3. ${ }^{(46)}$ If $g$ is a bounded function in $\mathscr{B}^{1}$, and if $f$ is in $\mathscr{B}^{q}$, then $g \cdot f$ is in $\mathscr{B}^{9}$.

Remark. The same result is true for $\mathscr{A}^{1}, \mathscr{A}^{q}$ instead of $\mathscr{B}^{1}, \mathscr{B}^{2}$.

ExAMPLE. The square $\mu^{2}$ of the Mobius function is bounded and is ${ }^{(47)}$ in $\mathscr{B}^{1}$. Hence for any function $f \in \mathscr{A}^{1}$ the mean value $M\left(n \mapsto \mu^{2}(n) f(n)\right)$ exists. If $f$ is multiplicative, this mean value is equal to

$$
\prod_{p}\left(1+\frac{f(p)-1}{p}-\frac{f(p)}{p^{2}}\right)
$$

Proof of Lemma 5.3. Given $\varepsilon>0$, choose $t_{1}, t_{2} \in \mathscr{B}$ with the properties $\left\|f-t_{1}\right\|_{q}<\varepsilon,\left\|g-t_{2}\right\|_{1}<\varepsilon$ and ${ }^{(48)}\left\|t_{2}\right\|_{\nu} \leqslant\|g\|_{u}$. Then

$$
\left\|g-t_{2}\right\|_{q} \leqslant 2^{q-1}\|g\|_{u}^{q-1}\left\|g-t_{2}\right\|_{1}
$$

and

$$
\begin{aligned}
\left\|f g-t_{1} t_{2}\right\|_{q} & \leqslant\left\|\left(f-t_{1}\right) g\right\|_{q}+\left\|t_{1}\left(g-t_{2}\right)\right\|_{q} \\
& \leqslant\|g\|_{u}^{q}\left\|f-t_{1}\right\|_{q}+2^{q-1}\|g\|_{u}^{2 q-1}\left\|q-t_{2}\right\|_{1}
\end{aligned}
$$

which is small.

(45) Daboussi proves: If $g \geqslant 0, \alpha \geqslant 1$ and $\beta \geqslant 1$, then $g^{\alpha} \in \mathscr{A}^{\beta}$ is equivalent with $g \in \mathscr{A}^{\alpha \beta}$.

(46) See Daboussi [8], III.8.

(47) Proof: $\mu^{2}$ and 1 are related. Apply Lemma 5.1, Corollary (2).

(48) This is possible, since for real-valued $t, t^{\prime} \in \mathscr{B}$ the functions $\min \left(t, t^{\prime}\right)$ and $\max \left(t, t^{\prime}\right)$ are in $\mathscr{B}$ again. 
LEMMA 5.4. If $f$ is in $\mathscr{B}^{1}$ and if $\|f\|_{q}$ is finite, where $q>1$, then $f$ is in $\mathscr{B}^{r}$ for any $r$ satisfying $1 \leqslant r<q$.

Proof. Without loss of generality assume that $f \in \mathscr{B}^{1}$ is real-valued. The truncated function

$$
f_{K}=\max \{-K, \min (f, K)\}
$$

is in $\mathscr{B}^{1}$, since $\max (c, f)$ and $\min \left(c^{\prime}, f\right)$ are in $\mathscr{B}^{1}$ again for any real constants $c, c^{\prime}$. The function $f_{K}$ is bounded, therefore $f_{K}$ is in $\mathscr{D}^{\prime}$ for any $l \geqslant 1$ (see Lemma 5.3). Given $r, 1 \leqslant r<q$, define $s, s^{\prime}$ by $s^{\prime}=q / r>1,1 / s+1 / s^{\prime}=1$. Then, applying Hölder's inequality,

$$
\begin{aligned}
\Delta(x) & :=\frac{1}{x} \sum_{n \leqslant x}\left|f(n)-f_{K}(n)\right|^{r} \leqslant \frac{1}{x} \sum_{\substack{n \leqslant x \\
|f(n)|>K}}|f(n)|^{r} \\
& \leqslant\left\{\frac{1}{x} \sum_{n \leqslant x}|f(n)|^{q}\right\}^{1 / s^{\prime}}\left\{\frac{1}{x} \sum_{\substack{n \leqslant x \\
|(n)|>X}} 1\right\}^{1 / s} .
\end{aligned}
$$

Obviously

$$
K^{q} \sum_{n \leqslant x,|\mathcal{S}(n)|>K} 1 \leqslant \sum_{n \leqslant x}|f(n)|^{q}
$$

and so

$$
\left\|f-f_{\mathbf{K}}\right\|_{r}^{r}=\underset{x \rightarrow \infty}{\limsup } \Delta(x) \leqslant\|f\|_{q}^{q / s^{r}}\left\{\frac{1}{K^{q}}\|f\|_{q}^{q}\right\}^{1 / s}<\varepsilon,
$$

if $K$ is sufficiently large. Therefore $f \in \mathscr{B}^{r}$.

\subsection{An upper estimate for sums over multiplicative functions}

LEMMA 5.5. If $f \geqslant 0$ is multiplicative and

$$
\sum_{p^{k} \leqslant y} f\left(p^{k}\right) \log p^{k} \leqslant c_{1} y
$$

then

$$
\sum_{n \leqslant x} f(n) \leqslant c_{2} x \exp \left(\sum_{p \leqslant x} \frac{f(p)-1}{p}+\sum_{p \leqslant x} \sum_{k \geqslant 2} \frac{f\left(p^{k}\right)}{p^{k}}\right),
$$

where $c_{2}$ only depends on $c_{1}$.

This lemma is well known (for the idea of the proof see for example Wirsing [81], Hilfssatz 2). The proof uses

$$
\text { - } \begin{aligned}
\sum_{n \leqslant x} f(n) \log n & =\sum_{n \leqslant x} f(n) \sum_{p^{k} \| n} \log p^{k}=\sum_{p^{k} \leqslant x} \log p^{k} \sum_{\substack{m \leqslant x p^{-k} \\
p \nmid m}} f(m) f\left(p^{k}\right) \\
& \leqslant \sum_{m \leqslant x} f(m) \sum_{p^{k} \leqslant x / m} f\left(p^{k}\right) \log p^{k} \leqslant c_{1} x \sum_{m \leqslant x} \frac{f(m)}{m}
\end{aligned}
$$


and

$$
\sum_{m \leqslant x} \frac{f(m)}{m} \leqslant \prod_{p \leqslant x}\left(1+\frac{f(p)}{p}+\ldots\right) \leqslant \exp \left(\sum_{p \leqslant x} \frac{f(p)}{p}+\sum_{p \leqslant x, k \geqslant 2} \frac{f\left(p^{k}\right)}{p^{k}}\right)
$$

and partial summation in connection with

$$
\sum_{p \leqslant x} 1 / p=\log \log x+O(1) .
$$

Example. Condition (5.5) is satisfied, if $f\left(p^{k}\right) \leqslant c_{3}$ for all primes $p$ and all $\dot{k} \geqslant 1$ is assumed, or if $f(p) \leqslant c_{3}$ and $f\left(p^{k}\right) \leqslant \lambda_{1} \lambda_{2}^{k}$ for $k \geqslant 2$, where $\lambda_{2}<2$ (see Wirsing [81], (28)).

\section{Proof for $\left(M_{1}\right)$}

In this section we give a sketch of the proof of

$$
\text { If } f \in \mathscr{E}_{q} \text { is multiplicative, } q \geqslant 1 \text {, then } f \in \mathscr{B}^{q} \text {, }
$$

following Schwarz-Spilker [72]. ${ }^{(49)(50)}$

Using Lemma 5.5 and Lemma 5.1 it is comparatively easy to prove

$$
\text { If } f \in \mathscr{E}_{q} \text { is multiplicative, then }\|f\|_{q}<\infty \text {. }
$$

The main step is to show

$$
\text { If } f \in \mathscr{E}_{1} \text { is multiplicative, then } f \in \mathscr{B}^{1} \text {. }
$$

Using relationship arguments (Lemma 5.1) assertion (6.2) is reduced to

LEMMA 6.1. If $f$ is strongly multiplicative, if the series

$$
\sum \frac{f(p)-1}{p} \text { and } \sum \frac{|f(p)-1|^{2}}{p} \text {. }
$$

are convergent, and if $|f(p)-1| \leqslant 1 / 4$, then $f \in \mathscr{B}^{1}$.

Lemma 6.1 is proved by Rényi's method: ${ }^{(51)}$ Approximate $f$ in $\|\cdot\|_{1}$ by

1491 The proof of Proposition 4, p. 333, is not correct as it stands. Moreover, for this proof the isomorphy of $x^{q}$ with a certain space $L^{q}\left(N^{*}, \mu\right)$ was used; this isomorphy is based either on Gelfand's theory of commutative Banach-algebras or on an explicit construction of a compactification $N^{*}$ of $N$ ( $N^{*}$ is the "maximal ideal space" in Gelfand's theory) - see SchwarzSpilker [70]. To avoid these difficulties, we give a variation of the proof of $\left(\mathrm{M}_{1}\right)$, simplifying the argument leading from (6.1) and (6.2) to $\left(\mathrm{M}_{1}\right)$.

(50) I have to thank Gabor Halász for drawing my attention to the problem of avoiding Proposition 4 from Schwarz-Spilker [72].

(51) Rényi [60], see also Schwarz-Spilker [71]. 
the strongly multiplicative functions $f_{K}^{*}$, defined by

$$
f_{K}^{*}(p)=\left\{\begin{array}{lll}
f(p), & \text { if } & p \leqslant K, \\
1, & \text { if } & p>K
\end{array}\right.
$$

$f_{K}^{*}$ is even $\bmod \prod_{p \leqslant K} p$, and so $f_{K}^{*}$ is in $\mathscr{B}$.

We aim at the relation

$$
\lim _{K \rightarrow \infty}\left\|f-f_{K}^{*}\right\|_{1}=0
$$

Define the strongly additive function

$$
w_{K}(n)=\sum_{p \mid n, p>K} \log f(p)
$$

and use the inequality $\left|e^{z}-1\right| \leqslant|z|+\left|z e^{z}\right|$ to obtain

$$
\Delta_{N}^{K}:=\frac{1}{N} \sum_{n \leqslant N}\left|f(n)-f_{K}^{*}(n)\right| \leqslant \frac{1}{N} \sum_{n \leqslant N}\left|f_{K}^{*}(n)\right|\left|w_{k}(n)\right|\left\{1+\left|e^{w_{k}(n)}\right|\right\} .
$$

Cauchy's inequality gives

$$
\Delta_{N}^{K} \ll\left\{\frac{1}{N} \sum_{n \leqslant N}\left|w_{K}^{2}(n)\right|\right\}^{1 / 2}\left(\left\|f_{K}^{*}\right\|_{2}+\|f\|_{2}\right)
$$

It is easy to show ${ }^{(52)}$ that the norms $\|f\|_{2}$ and $\left\|f_{K}^{*}\right\|_{2}$ are bounded (uniformly in $K$ ). Abbreviating $(1 / N) \sum_{n \leqslant N}\left|w_{K}^{2}(n)\right|$ by $\Omega_{K, N}$, we obtain

$$
\Omega_{K, N} \leqslant \frac{2}{N} \sum_{n \leqslant N}\left|w_{K}(n)-\sum_{p \leqslant N} \frac{w_{K}(p)}{p}\right|^{2}+2\left|\sum_{p \leqslant N} \frac{w_{K}(p)}{p}\right|^{2}=\Omega_{K, N}^{(1)}+\Omega_{K, N}^{2} .
$$

Put $w(p)=\log f(p)=\log r(p)+i \vartheta(p)$; by the Turán-Kubilius inequality, ${ }^{(53)}$ we get.

$$
\Omega_{K, N}^{(1)} \ll \sum_{K<p \leqslant N} \frac{1}{p}\left(\log ^{2} r(p)+\vartheta^{2}(p)\right)
$$

and

$$
\Omega_{K, N}^{(2)} \ll\left(\sum_{K<p \leqslant N} \frac{\log r(p)}{p}\right)^{2}+\left(\sum_{K<p \leqslant N} \frac{\vartheta(p)}{p}\right)^{2}
$$

(52) Use Lemma 5.5 .

(53) If $w$ is strongly additive, then

$$
\frac{1}{N} \sum_{n \leqslant N}\left|w(n)-\sum_{p \leqslant N} \frac{w(p)}{p}\right|^{2} \leqslant C_{1} \sum_{p \leqslant N} \frac{|w(p)|^{2}}{p},
$$

where $C_{1}$ is an absolute constant. For a simple proof see Elliott [18]. 
It is not difficult to prove the convergence of the four series occurring in (6.5.1) and (6.5.2), by use of the assumptions (6.3). Hence $\Omega_{K, N}^{(1)}$ and $\Omega_{K, N}^{(2)}$ tend to zero for $K \rightarrow \infty$, and Lemma 6.1 is proved. $f^{\S}$ by

In order to show that $(6.1)$ and $(6.2)$ imply $\left(\mathrm{M}_{1}\right)$ we define the function

$$
f^{\xi}(n)=\prod_{p^{k} \| n,\left|f\left(p^{k}\right)\right| \leqslant K} f\left(p^{k}\right) .
$$

Then

$$
f^{\S} \text { is near } f \text { with respect to }\|\cdot\|_{q} \text {, if } K \text { is large, }
$$

and

$$
f^{\S} \text { is in } \mathscr{B}^{q} \text { for any } K \text {. }
$$

Obviously, (6.6) and (6.7) imply $f \in \mathscr{B}^{9}$.

Concerning (6.6), calculate

$$
\Sigma(x):=\sum_{n \leqslant x}\left|f(n)-f^{\xi}(n)\right|^{q}=\sum_{n \leqslant x}\left|f^{\S}(n)\right|^{q}\left|\prod_{\substack{p^{k} \| n \\\left|f\left(p^{k}\right)\right|>K}} f \cdot\left(p^{k}\right)-1\right|^{q} .
$$

The second factor is zero if there is no prime-power $p^{k} \| n$, for which $\left|f\left(p^{k}\right)\right|>K$. Using the inequality $|\alpha-1|^{q} \leqslant 2^{q}|\alpha|^{q}$ (valid, if $|\alpha| \geqslant 1$ ), we obtain

$$
\Sigma(x) \leqslant 2^{q} \sum_{n \leqslant x}^{(*)}|f(n)|^{q},
$$

where the condition $(*)$ means that there exists a prime-power $p^{k}$ exactly dividing $n$ for which $\left|f\left(p^{k}\right)\right|>K$. Since

$$
\begin{aligned}
\sum_{n \leqslant x}^{(*)}|f(n)|^{q} & \leqslant \sum_{\substack{p \\
\left|S\left(p^{k}\right)\right|>K}} \sum_{k \geqslant 1} \sum_{\substack{m \leqslant x \cdot p^{-k} \\
p \nmid m}}|f(m)|^{q}\left|f\left(p^{k}\right)\right|^{q} \\
& \leqslant C x\left\{\sum_{p,|f(p)|>K} \frac{|f(p)|^{q}}{p}+\sum_{\substack{p \\
\left|f\left(p^{k}\right)\right|>K}} \sum_{k \geqslant 2} \frac{\left|f\left(p^{k}\right)\right|^{q}}{p^{k}}\right\}
\end{aligned}
$$

the convergence of $S_{2}^{\prime \prime}(f)$ and $S_{3}(f)$ [for the definition of these series see Section 1.4] implies that the curly bracket $\{\ldots\}$ is small, if $K$ is large.

Since $f^{*}$ is in $\mathscr{B}^{1}$ according to (6.2), assertion (6.7) will follow from Lemma 5.4, if

$$
\left\|f^{8}\right\|_{r}<\infty \quad \text { for some } r>q
$$

is shown. By Lemma 5.5

$$
\sum_{n \leqslant x}\left|f^{k}(n)\right|^{r} \leqslant c_{2} x \exp \left\{\sum_{\substack{p \leqslant x \\|f(p)| \leqslant K}} \frac{|f(p)|^{r}-1}{p}+\sum_{p \leqslant x_{1}, k \geqslant 2,\left|f\left(p^{k}\right)\right| \leqslant K} \sum^{-k}\left|f\left(p^{k}\right)\right|^{r}\right\} .
$$


The second (double-) sum in the exponent is obviously

$$
\leqslant \sum_{p} \sum_{k \geqslant 2} p^{-k}\left|f\left(p^{k}\right)\right|^{q} K^{r-q}=K^{r-q} S_{3}(f)<\infty
$$

(see Section 1.4). The convergence of $S_{2}^{\prime \prime}(f)$ and of $S_{2}^{\prime}(f)$ reduces the problem of showing the convergence of the series $\sum_{p \leqslant x,|S(p)| \leqslant k} p^{-1}\left(|f(p)|^{r}-1\right)$ to the assertion that

$$
\sum_{\substack{p \\ p}} p^{-1}\left(|f(p)|^{r}-1\right) \text { is convergent. }
$$

Since (in $1 / 2 \leqslant y \leqslant 3 / 2$ )

$$
y^{r}-1=r(y-1)+O\left(|y-1|^{2}\right),
$$

the convergence of (6.9) follows from the convergence of $S_{2}^{\prime}(f)$ and of

$$
\sum_{|f(p)| \leqslant 3 / 2} p^{-1}\{|f(p)|-1\}
$$

the convergence of the series (6.10) is easily deduced from the assumption $f \in \mathscr{E}_{1}$ (see Schwarz-Spilker [72], proof of Lemma 2.2).

Thus the proof of $\left(\mathrm{M}_{1}\right)$ is complete.

\section{Proof for $\left(\mathrm{M}_{2}\right)$}

We are going to sketch a proof ${ }^{(54)}$ for

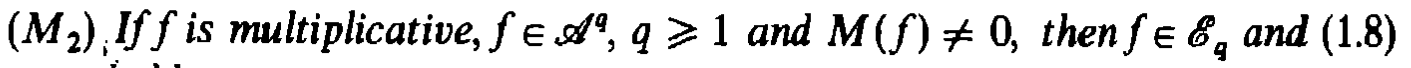
holds.

We begin with the special case $q=2$, assuming 'without loss of generality that $f$ is strongly multiplicative. The case $q=2$ was treated by Daboussi and Delange [11], and we follow their method with one short-cut, namely:

To prove

$$
\sum \frac{1}{p}|f(p)-1|^{2}<\infty
$$

we do not use the so-called "dual of the Turán-Kubilius inequality" as Elliott [17] and Daboussi-Delange did. Instead of this we calculate the Ramanujan coefficients

$$
a_{p}^{*}:=\frac{1}{M(f)} a_{p}(f)=\frac{M\left(f c_{p}\right)}{\varphi(p) M(f)}=\frac{f(p)-1}{p}+O\left(\frac{1+|f(p)|^{2}}{p^{2}}\right)
$$

(54) Following Schwarz-Spilker [73]. 
for primes $p$; this is rather easy. The Ramanujan sums $c_{r}$ being orthogonal, Bessel's inequality gives

$$
\infty>\frac{1}{|M(f)|^{2}}\|f\|_{2}^{2} \geqslant \sum_{p}\left|a_{p}^{*}\right|^{2} \varphi(p)=\sum_{p} \frac{1}{p}|f(p)-1|^{2}+O(1) .
$$

Remark. In fact this method proves: If for multiplicative $f$ the mean value $M(f)$ exists and is non-zero, and if $\|f\|_{2}<\infty$, then $f \in \mathscr{E}_{2}$ and (1.8) holds.

The idea for the general case $q \geqslant 1$ is to apply relationship arguments but how to begin with?

The assumption $f \in \mathscr{A}^{q}, M(f) \neq 0$ implies $|f| \in \mathscr{A}^{q}$ and $M(|f|) \neq 0$, and hence by Lemma 5.2

$$
|f|^{q / 2} \in \mathscr{A}^{2}
$$

A short calculation gives

$$
M\left(|f|^{q / 2}\right) \neq 0 .
$$

Applying the results obtained already for the special case $q=2$ to $|f|^{\mid / 2}$, we obtain the convergence of the series

$$
\begin{gathered}
\sum_{p} p^{-1}\left(|f(p)|^{q / 2}-1\right) \\
\sum_{p} p^{-1}\left(|f(p)|^{q / 2}-1\right)^{2}
\end{gathered}
$$

and

$$
\sum_{p} \sum_{k \geqslant 2} p^{-k}\left|f\left(p^{k}\right)\right|^{q}
$$

The convergence of (7.2.2) implies

$$
\sum_{p,\left|\int(p)\right|<1 / 2} \frac{1}{p}<\infty \quad \text { and } \quad \sum_{p,|f(p)|>3 / 2} \frac{1}{p}<\infty
$$

and therefore relationship arguments can be applied. Define (for a suitable $\left.P_{0}\right)$

$$
f\left(p^{k}\right)= \begin{cases}f\left(p^{k}\right), & \text { if } p \leqslant P_{0}, \\ f(p), & \text { if } p>P_{0}, k=1,1 / 2<|f(p)| \leqslant 3 / 2, \\ 0, & \text { otherwise. }\end{cases}
$$

Then $\tilde{f}=f * h$, where $\sum n^{-1}|h(n)|<\infty$, and so $\tilde{f} \in \mathscr{A}^{1}$ and $M(\tilde{f}) \neq 0$. Our aim " $\tilde{f} \in \mathscr{A}^{2}$ " need not be true due to the primes $p \leqslant P_{0}$.

Denote by $\mu_{K}^{2}$ the characteristic function of the set of $K$-free integers. This function is in $\mathscr{B}^{1}$ (and even in $\mathscr{B}$ for any $r \geqslant 1$, but this fact is not needed here); this follows for example from Lemma 5.1 or from the results of Section 6 . 
Therefore

$$
f^{\sim \sim}:=\mu_{\mathrm{K}}^{2} \tilde{f}
$$

is in $\mathscr{A}^{1}$, according to a simple result from the theory of almost-periodic functions. ${ }^{(55)}$ If $K$ is chosen sufficiently large (this depends on $P_{0}$ ), then $M\left(f^{\sim}\right) \neq 0$. A short calculation ${ }^{(56)}$ gives

$$
\left\|f^{\sim \sim}\right\|_{2}<\infty \text {. }
$$

Therefore, by the results in the special case $\| f^{\sim \sim \|_{2}}<\infty$, we obtain the convergence of

$$
\sum_{p,|f(p)| \leqslant 3 / 2} \frac{1}{p}|f(p)-1|^{2}
$$

and the convergence of

$$
\sum_{\substack{p \\ 1 / 2<|S(p)| \leqslant 3 / 2}} \frac{1}{p}(f(p)-1) ;
$$

using (7.2.2) and (7.2.1) and again (7.3) we obtain the convergence of

$$
\sum_{p} \frac{1}{p}(f(p)-1) \quad \text { and } \quad \sum_{\substack{p \\|f(p)|^{p}>3 / 2}} \frac{1}{p}|f(p)|^{q},
$$

and we are done.

\section{Problems}

(8.1) It is known that the Ramanujan expansion

$$
f(n)=\sum_{r=1}^{\infty} a_{r}(f) c_{r}(n)
$$

is pointwise convergent, if $f$ is an arithmetical function in $\mathscr{B}^{q}, q>1$, which is additive or is multiplicative with mean value $M(f) \neq 0$. A. Hildebrand [36] showed the convergence of (8.1) for any $f$ in $\mathscr{B}^{4}$.

Can one give a "simple" summability method summing the series (8.1) for "many" $f$ in $\mathscr{B}^{q}$ ?

(8.2) It is easily possible to characterize $\mathscr{D}^{2}$ by translation properties: $f \in \mathscr{D}^{u}$ if and only if for any $\varepsilon>0$ there exists a $k \in N$ such that $\left\|f-f_{(k)}\right\|_{u}<\varepsilon$, where $f_{(k)}(n)=f$ (g.c.d. $\left.(k, n)\right)$.

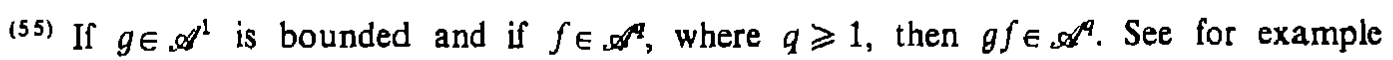
Daboussi [8], III.8, or Lemma 5.3.

(56) See Lemma 5.5. 
Is it possible to give a similar characterization for functions in $\mathscr{B}^{q}$ ?

(8.3) Is it possible to characterize functions in $\mathscr{O}^{9}$ by some "structural" or "number-theoretical" properties?

(8.4) According to Gelfand's theory of commutative Banach algebras $\mathscr{A}^{\mu} \cong \mathscr{C}\left(N^{* *}\right), \mathscr{D}^{u} \cong \mathscr{C}\left(N^{* * *}\right)$. Is it possible to give a "reasonable"explicite description of $N^{* *}$ and $N^{* * *}$, as it is possible for $N^{*}$, where $\mathscr{B}^{\mu} \cong \mathscr{C}\left(N^{*}\right)$ ?

(8.5) What can be said on the factor spaces $\mathscr{A}^{q} / \mathscr{B}^{q}, \mathscr{D}^{q} / \mathscr{B}^{q}$ ? 5.1).

(8.6) Give a quantitative version of the relationship theorem (Lemma

(8.7) Try to deduce a relationship theorem for arithmetical functions, which need not to be multiplicative, but are in some sense "close" to multiplicative ones.

EXAMPLE. Denote by $\alpha, \beta$ the characteristic functions of the sets $A, B \subset N$. Assume there is a direct decomposition $N=A \times B$; this is equivalent with $1=\alpha * \beta$. The functions $\alpha, \beta$ need not be multiplicative, however there is some kind of multiplicative structure. Saffari [62] proved: If

$$
\sum_{n=1}^{\infty} n^{-1} \beta(n)<\infty
$$

then $M(\alpha)$ exists.

An elegant proof of Saffari's result was given by $H$. Daboussi [7]. J. Spilker (unpublished) proved $\alpha \in \mathscr{B}^{1}$, if $(*)$ is true.

If $\alpha$ were multiplicative, then the existence of $M(\alpha)$ would immediately follow from the relationship theorem.

(8.8) If $f$ is multiplicative in $\mathscr{B}^{2}$ with mean value $M(f) \neq 0$, and if infinitely many Ramanujan coefficients $a_{r}(f)$ are non-zero, then the Hankel determinants

$$
\mathscr{H}_{n}=\operatorname{det}\left[\begin{array}{llll}
f(1) & f(2) & & f(n) \\
f(2) & f(3) & \ldots & f(n+1) \\
\ldots \ldots & \ldots \ldots \ldots \ldots \ldots \ldots \ldots \\
f(n) & f(n+1) & \ldots & f(2 n-1)
\end{array}\right]
$$

are non-zero for infinitely many $n$. This follows from the result on noncontinuability in Section 4 in combination with a theorem of Kronecker from complex function theory. ${ }^{(57)}$

Is it possible to prove this result more directly?

(57) See for example Bieberbach [3], Sec. 6.2. 


\section{References}

[1] M. Barban, The "large sleven method and its applications to number theory, Uspekhi Mat. Nauk 21 (1966), 51-102; transl. in Russian Math. Surveys 21 (1966), 49-104.

[2] A. S. Besicovitch, Almost periodic functions, Cambridge 1932.

[3] L. Bieberbach, Analytische Fortsetzung, Berlin-Göttingen-Heidelberg 1955.

[4] N. G. de Bruijn, Bijna periodieke multiplicatieve functies, Nieuw Arch Wisk. 22 (1943), 81-95.

[5] E. Cohen, $A$ class of arithmetical functions, Proc. Nat. Acad. Sci. USA 41 (1955), 939-944.

[6] H. Daboussi, Sur les fonctions multiplicatives ayant une valeur moyenne non nulle, Preprint Université de Paris-Sud, 1978, 1-21. Bull. Soc. Math. France 109 (1981), 183-205.

[7] -, On the density of direct factors of the set of positive integers, J. London Math Soc. (2) 19 (1979), 21-24.

[8] -, Caractérisation des fonctions multiplicatives p.p. $B^{d}$ a spectre non vide, Ann. Inst. Fourier (Grenoble) 30 (1980), 141-166.

[9] -, On the limiting distribution of non negative additive functions, Comp. Math. 43 (1981), 101-105.

[10] H. Daboussi and H. Dela nge, Quelques propriétés des fonctions multiplicatives de module au plus égal à 1, C. R. Acad. Sci. Paris 278 (1974), A 657-660.

[11] -, -, On a theorem of P. D. T. A. Elliott on multiplicative functions, J. London Math. Soc. 14 (1976), 345-356.

[12] H. Delange, Sur les fonctions arithmétiques multiplicatives, Ann. Sci. École Norm. Sup. 78 (1961), 273-304.

[13] -, Application de la méthode du crible à Pétude des valeurs moyennes de certaines fonctions arithmétiques, Séminaire Delange-Pisot (Théorie des nombres), 3e année, 1961/62, no. 16, 10 pages.

[14] -, On a class of multiplicative arithmetical functions, Scripta Math. 26 (1963), 121-141.

[15] - A remark on multiplicative functions, Bull. London Math. Soc. 2 (1970), 183-185.

[16] -, Sur les fonctions multiplicatives de plusieurs entiers, L'Enseignement Math. 16 (1970), 219-246.

[17] P. D. T. A. Elliot t, $A$ mean-value theorem for multiplicative functions, Proc. London Math. Soc. (3) 31 (1975), 418-438.

[18] -, The Turán-Kubilius inequality, Proc. Amer. Math. Soc. 65 (1977), 8-10.

[19] -, On the differences of additive arithmetic functions, Mathematika 24 (1977), 153-165.

[20] -, Probabilistic Number Theory, Vol. I, Vol. Il, Springer Grundlehren, New YorkHeidelberg-Berlin 1979, 1980.

[21] -, Mean value theorems for multiplicative functions bounded in mean $\alpha$-power, $\alpha>1, \mathrm{~J}$. Austral. Math. Soc. Ser. A 29 (1980), 177-205.

[22] -, High-power analogues of the Turán-Kubilius inequality, and an application to number theory, Canad. J. Math. 32 (1980), 893-907.

[23] P. Erd6s, On the distribution function of additive functions, Ann. of Math. (2) 47 (1946), 120.

[24] -, Some asymptotic formulas for multiplicative functions, Bull. Amer. Math. Soc. 53 (1947),

536-544.
[25] On the sum $\sum_{k=1}^{x} d(f(k))$, J. London Math. Soc. 27 (1951), 7-15.

[26] $\mathrm{P}$. Erdos and A. Rényi, On the mean value of nonnegative multiplicative number-theoretical functions, Michigan Math. J. 12 (1965), 321-338.

[27] P. Erdoss, B. Saffari and R. C. Vaughan, On the asymptotic density of sets of integers, II, J. London Math. Soc. (2) 19 (1979), 17-20.

[28] G. Halász, Uber die Mittelwerte multiplikativer zahlentheoretischer Funktionem, Acta Math. Sci. Hung. 19 (1968), 365-403. 
[29] G. Halász, Uber die Konvergenz multiplikativer zahlentheoretischer Funktionen, Studia Sci. Math. Hungar. 4 (1969), 171-178.

[30] -, On the distribution of additive and the mean-values of multiplicative arithmetic functions, ibid. 6 (1971), 211-233.

[31] P. Harlmann and A. Wintner, On the almost periodicity of additive number-theoretical functions, Amer. J. Math. 62 (1940), 753-758.

[32] E. Heppner, Uber benachbarte multiplikative zahlentheoretische Funktionen mehrerer Variablen, Archiv Math. 35 (1980), 454-460.

[33] -, Uber Mittelwerte multiplikativer zahlentheoretischer Funktionen mehrerer Variablen, Monatsh. Math. 91 (1981), 1-9.

[34] -, Uber Mittelwerte multiplikativer zahlentheoretischer Funktionen, Ann. Univ. Sci. Budapest 25 (1982), 85-96.

[35] E. Heppner und W. Schwarz, Benachbarte multiplikative Funktionen, Studies in Pure Mathematics. Den Andenken Paul Turán's gewidmet, 1983, 323-336.

[36] A. Hildebrand. Uher die punktweise Konvergenz von Ramanujan-Entwicklungen zahlentheoretischer Funktionen, Acta Arith. 44 (1984), 109-140.

[37] A. Hildebrand und J. Spilker, Charakterisierung der additiven, fast-geraden Funktionen, Manuscripta Math. 32 (1980), 213-230.

[38] K. H. Indlek ofer, $A$ mean value theorem for multiplicative functions, Math. Z. 172 (1980), 255-271.

[39] -. Properties of uniformly summable multiplicative functions, Preprint 1980.

[40] -, Some results on the behaviour of additive and multiplicative functions, Preprint Nov. 1980.

[41] -, Remark on a theorem of G. Halász, Archiv Math. 36 (1981), 145-151.

[42] -, Some remarks on almost-even and almost-periodic functions, ibid. 37 (1981), 353-358.

[43] -, Limiting distributions and mean-values of arithmetical functions, J. Reine Angew. Math. 328 (1981), 116-127.

[44] A. E. Ingham, On absolutely convergent Dirichlet series, Studies in Math. Analysis and Related Topics. Essays in Honor of G. Pólya, 156-164, Stanford Calif. 1962.

[45] E. R. van Kampen, On uniformly almost periodic multiplicative and additive functions, Amer. J. Math. 62 (1940), 627-634.

[46] J. Knoplmacher, Abstract Analytic Number Theory, Amsterdam-Oxford 1975.

[47] -, Fourier analysis of arithmetical functions, Annali Mat. Pura Appl. (IV) 109 (1976), $177-$ 201 .

[48] B. V. Levin and N. M. Timofeev, Sums of multiplicative functions, Dokl. Akad. Nauk SSSR 193 (1970), 992-995.

[49] L. Lucht, Uber benachbarte multiplikative Funktionem, Archiv Math. 30 (1978), 40-48.

[50] -, Mittelwerte zahlentheoretischer Funktionen und lineare Kongruenzsysteme, J. Reine Angew. Math. 306 (1979), 212-220.

[51] -, Mittelwerte multiplikativer Funktionen auf Linearformen, Archiv Math. 32 (1979), 349355.

[52] -, Power series with multiplicative coefficients, Math. Z. 177 (1981), 359-374.

[53] L. Lucht and F. Tuttas, Mean-values of multiplicative functions and natural boundaries of power series with multiplicative coefficients, J. London Math. Soc. (2) 19 (1979), 25-34.

[54] E. Lukacs, Characteristic Functions, 2nd ed., Griffin, London 1970, 350 pages.

[55] D. J. Newman, $A$ simple proof of Wener's (1/f)-theorem, Proc. Amer. Math. Soc. 48 (1975), 264-265.

[56] A. Parson and J. Tull, Asymptotic behavior of multiplicative functions, J. Number Theory 10 (1978), 395-420.

[57] G. Pólya, Úber Potenzreihen mit ganzzahligen Koeffizienten, Math. Ann. 77 (1916), 497513. 
[58] G. Pólya, Sur les séries entières à coefficients entiers, Proc. London Math. Soc. (2) 21 (1921), 22-38.

[59] A. Rényi, On a theorem of $P$. Erdös and its application in information theory, Mathematica (Cluj) 24 (1959), 341-344.

[60] -, A new proof of a theorem of Delange, Publ. Math. Debrecen 12 (1965), 323-329.

[61] L. Rubel and K. Stolarsky, Subseries of the power series for $e^{x}$, Amer. Math. Monthly 87 (1980), 371-376.

[62] B. Saffari, On the asymptotic density of sets of integers, J. London Math. Soc. (2) 13 (1976), 475-485.

[63] W. Schwarz, A remark on multiplicative functions, Bull. London Math. Soc. 4 (1972) 136140.

[64] -, Eine weitere Bemerkung über multiplikative Funktionen, Coll. Math. 28 (1973), 81-89.

[65] -, Ramanujan-Entwicklung stark multiplikativer Funktionen, J. Reine Angew. Math. 262/263 (1973), 66-73.

[66] - Uber die Ramanujan-Entwicklung multiplikativer Funktionen, Acta Arith. 27 (1975), 269279.

[67] -, Aus der Theorie der zahlentheoretischen Funktionen, Jahresber. Deutsch. Math.-Verein. 78 (1976), 147-167.

[68] -, Some applications of Elliott's mean-value theorem, J. Rejne Angew. Math. 307/308 (1979), 418-423.

[69] -, Fourier--Rumanujan-Entwicklungen zahlentheoretischer Funktionen und Anwendungen, Festschrift der Wissenschaftlichen Gesellschaft, Franz Steiner Verlag, Wiesbaden 1981, 399415.

[70] W. Schwarz und J. S pilker, Eine Anwendung des Approximationssatzes von WeierstrassStone auf Ramanujan-Summen, Nieuw Arch. Wisk. (3) 19 (1971), 198-209.

[71] -, -, Mean-values and Ramanujan expansions of almost even functions, Coll. Math. Soc. János Bolyai, Debrecen 1974 (1976), 315-357.

[72] -, -, Remarks on Elliott's Theorem on Mean-Values of Multiplicative Functions; in: Recent Progress in Analytic Number Theory, Vol. I, (Durham) Academic Press, London (1981), pp. 325-339.

[73] -, -, Eine Bemerkung zur Charaklerisierung der fast-periodischen multiplikativen zahlentheoretischen Funktionen mit von Null verschiedenem Mittelwert, Analysis 3 (1983), 205-216.

[74] J. S pilker und W. Schwar2, Wiener-Lévy-Sätze für absolut konvergente Reihen, Archiv Math. 32 (1979), 267-275.

[75] G. Szegö, Ubber Potenzreihen mit endlich vielen verschiedenen Koeftizienten, Sitzungsberichte d. Preuss. Akad. Wiss., Math.-Phys. Klasse 1922, 88-91.

[76] M. I. Tu lyagano va, A certain extension of a theorem of Halász, Izv. Akad. Nauk USSR, Ser. Fiz.-Mat. Nauk 14 (5) (1970), 35-40.

[77] F. Tuttas, Uber die Entwicklung multiplikativer Funktionen nach Ramanujan-Summen, Acta Arith. 36 (1980), 257-270.

[78] R. Warlimont, Die Entwicklung zahlentheoretischer Funktionen nach Ramanujan-Summen, Acta Arith. 42 (1983), 111-120.

[79] H. Weyl, Uber die Gleichverteilung von Zahlen modulo Eins, Math. Ann. 77 (1916), 313352.

[80]. A. Wintner, Eratosthenian averages, Baltimore 1943.

[81] E. Wirsing, Das asymptotische Verhalten von Summen über multiplikative Funktionen, Math. Ann. 143 (1961), 75-102.

[82] -, Das asymptotische Verhalten von Summen über multiplikative Funktionen, II, Acta Math. Acad. Sci. Hung. 18 (1967), 411-467.

[83] -, A characterization of $\log n$ as an additive arithmetic function, Symposia Math. dell' 
Istituto Nazionale di Alta Matematica, Roma, Vol. IV, 45-57, Academic Press, London, New York 1970.

[84] E. Wirsing, Additive functions with restricted growth on the numbers of the form $p+1$, Acta Arith. 37 (1980), 345-357.

[85] -, Additive and completely additive functions with restricted growth; in: Recent Progress in Analytic Number Theory (Symp. Durham, 1979), Vol. II, Academic Press, London 1981, pp. 231-280.

[86] D. Wolke, Multiplikative Funktionen auf schnell wachsenden Folgem, J. Reine Angew. Math. 251 (1971), 54-67.

Aded in proof (Sept. 25, 1984): In the proof of the result of Schwarz and Spilker[72] quoted on p. 469 a definitely incorrect result (Proposition 4) is used. However, for the proof of the quoted result Proposition 4 is only needed for multiplicative functions, and for such functions Proposition 4 is true. We refer to

W. Schwarz A correction to "Remarks on Elliott's Theorem on Mean-Values of Multiplicative Functions" (Durham 1979/1981) and Some remarks on almost-even numbertheoretical functions (Proceedings of the Conference at CRM Luminy, 1983, to appear),

where, among other things, Proposition 4 from [72] is proved for multiplicative functions.

\section{Presented to the Semester}

Elementary and Analytic Theory of Numbers

September 1-November 13, 1982

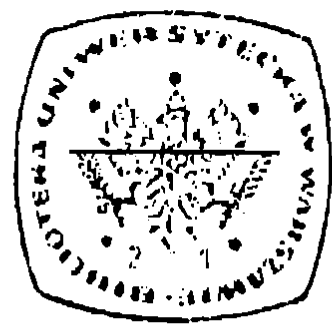

